\title{
Towards a weakly supervised framework for 3D point cloud object detection and annotation
}

\section{Journal Article}

Author(s):

Meng, Qinghao; Wang, Wenguan (1); Zhou, Tianfei; Shen, Jianbing; Jia, Yunde; Van Gool, Luc

Publication date:

2022-08

Permanent link:

https://doi.org/10.3929/ethz-b-000519405

Rights / license:

In Copyright - Non-Commercial Use Permitted

Originally published in:

IEEE Transactions on Pattern Analysis and Machine Intelligence 44(8), https://doi.org/10.1109/TPAMI.2021.3063611 


\title{
Towards A Weakly Supervised Framework for 3D Point Cloud Object Detection and Annotation
}

\author{
Qinghao Meng, Wenguan Wang, Member IEEE, Tianfei Zhou, \\ Jianbing Shen, Senior Member IEEE, Yunde Jia, Member IEEE, Luc Van Gool, Member IEEE
}

\begin{abstract}
It is quite laborious and costly to manually label LiDAR point cloud data for training high-quality 3D object detectors. This work proposes a weakly supervised framework which allows learning 3D detection from a few weakly annotated examples. This is achieved by a two-stage architecture design. Stage- 1 learns to generate cylindrical object proposals under inaccurate and inexact supervision, obtained by our proposed BEV center-click annotation strategy, where only the horizontal object centers are click-annotated in bird's view scenes. Stage-2 learns to predict cuboids and confidence scores in a coarse-to-fine, cascade manner, under incomplete supervision, i.e., only a small portion of object cuboids are precisely annotated. With KITTI dataset, using only 500 weakly annotated scenes and 534 precisely labeled vehicle instances, our method achieves $86-97 \%$ the performance of current top-leading, fully supervised detectors (which require 3, 712 exhaustively annotated scenes with 15, 654 instances). More importantly, with our elaborately designed network architecture, our trained model can be applied as a 3D object annotator, supporting both automatic and active (human-in-the-loop) working modes. The annotations generated by our model can be used to train 3D object detectors, achieving over $95 \%$ of their original performance (with manually labeled training data). Our experiments also show our model's potential in boosting performance when given more training data. The above designs make our approach highly practical and open-up opportunities for learning 3D detection at reduced annotation cost.
\end{abstract}

Index Terms-3D object detection, 3D annotation, Weakly supervised learning, Cascade inference, Autonomous driving

\section{INTRODUCTION}

$\mathrm{O}$ VER the past several years, extensive industry and research efforts have been dedicated to autonomous driving. Significant progress has been made in key technologies for innovative autonomous driving functions, with 3D object detection being one representative example.

Almost all recent successful 3D object detectors are built upon fully supervised deep learning techniques. They provide various solutions that tackle the problem by exploring information from monocular images [2], [3], stereo images [4], point clouds [5]-[8], or multi-modal signals [9], [10]; give insight into point cloud representation, introducing techniques such as voxelization [11], [12] and point-wise operation [13]; and greatly advanced the state-of-the-arts. However, these methods require large-scale, precisely-annotated 3D data to reach performance saturation and avoid overfitting.

Unfortunately, meeting such data requirements involves an astonishing amount of manual work, as it takes hundreds of hours to annotate just one hour of driving data. The end result is that a corpus of $3 \mathrm{D}$ training data is not only costly to create, but also typically limited in size and variety. This further prevents the applicability of fully supervised solutions

- Q. Meng and Y. Jia are with School of Computer Science, Beijing Institute of Technology, China. (Email: \{mengqinghao, jiayunde\}@bit.edu.cn)

- W. Wang, T. Zhou, and L. Van Gool are with ETH Zurich, Switzerland. (Email: \{wenguan.wang, ztfei.debug\}@gmail.com, vangool@vision.ee.ethz.ch)

- J. Shen is with both School of Computer Science, Beijing Institute of Technology, China and Inception Institute of Artificial Intelligence, UAE. (Email: shenjianbingcg@gmail.com)

- A preliminary version of this work has appeared in ECCV 2020 [1].

- Corresponding author: Wenguan Wang

- Our codes and annotated data are available in https://github.com/ hlesmgh/WS3D. in real-world settings. In short, 3D object detection systems will never become available by only integrating more and better technologies; the demand for massive, high-quality yet expensive labeled data has become one of the biggest challenges faced by 3D object detection system developers.

In order to promote the deployment of 3D object detection systems, it is necessary and urgent to reduce the heavy data annotation burden. Unfortunately, this essential issue has not yet received due attention. To this end, we propose a weakly supervised method that learns 3D object detection from less training data, with more easily acquired annotations. Specifically, our model has two main stages. Stage-1 learns to predict the object centers on the $(x, z)$-plane and identify foreground points. The training in this stage only requires a small set of weakly annotated bird's eye view (BEV) maps, where the horizontal object centers are roughly labeled (Fig. 1(b)). Such inexact and inaccurate supervision greatly saves annotation efforts. Since the height information is missing in BEV maps, we generate a set of cylindrical proposals whose extent along the $y$-axis is unlimited. Then, Stage-2 learns to estimate 3D parameters from these proposals and predict corresponding confidence scores with a cascade network structure. The learning paradigm in this stage is achieved by a few, precisely-annotated object instances as incomplete supervision (Fig. 1(c)), in contrast to prior arts [5], [14], which consume massive, exhaustively-labeled scenes (full ground-truth labels, Fig.1(a)).

Our weakly supervised framework has two appealing characteristics. First, it learns 3D object detection by making use of a small amount of weakly-labeled BEV data, mixed with precisely-annotated object instances. Specifically, the weak supervision from the BEV maps is in the form of click 

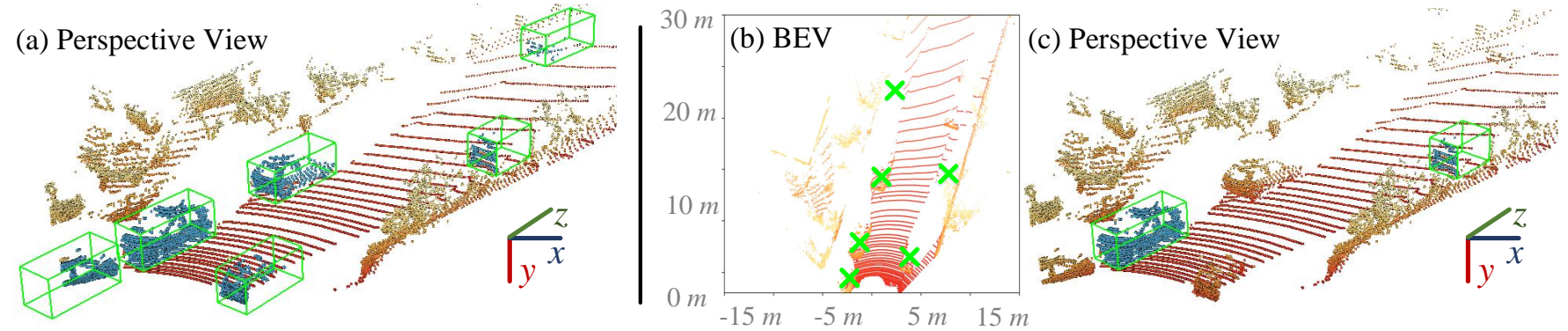

Fig. 1. Comparison between the full supervision used in prior arts (a) and our inaccurate, inexact (b) and incomplete (c) supervision. Previous fully supervised methods are trained from massive, exhaustively-labeled scenes (3,712 precisely annotated scenes with 15,654 vehicle instances), while our model uses only 500 weakly annotated scenes with center-annotated BEV maps as well as 534 precisely labeled vehicle instances.

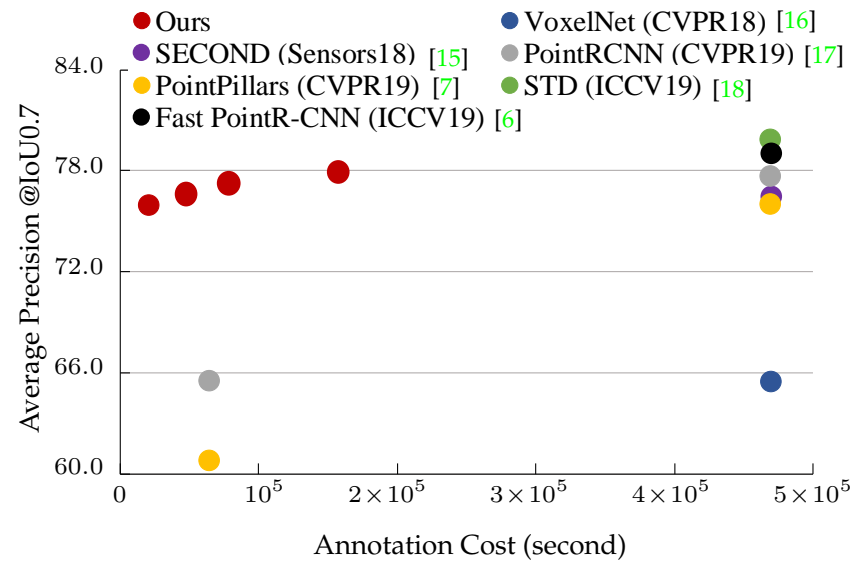

Fig. 2. Performance vs. annotation cost, tested on KITTI [19] val set (Car), under the moderate regime. Compared with current fully supervised 3D detectors, our model yields promising results with a far lower annotation demand (see §6.2). More importantly, when providing more annotations, our model shows gradually improved performance.

annotations of horizontal object centers. This enables much faster and easier data labeling compared to strong supervision requiring cuboids to be elaborately annotated on point clouds (about $40 \sim 50 \times$ faster; see $\S 3$ ). For the small set of well-annotated object instances, we only label $25 \%$ of objects in the weakly-labeled scenes, which is about $3 \%$ of the supervision used in current leading models. Such a weakly supervised 3D object detection paradigm not only provides the opportunity to reduce the strong supervision requirements in this field, but also introduces potential commercial benefits. Experiments on KITTI dataset [19] demonstrate that our detector is able to generate promising results over different classes. Specifically, experiments on the Car class (§6.2) show that, using only 500 weakly annotated scenes and 534 precisely labeled vehicle instances, we achieve $86-97 \%$ of the performance of fully supervised state-of-thearts (which require 3,712 precisely annotated scenes with 15,654 vehicle instances). When using more training data, our performance is further boosted (Fig. 2, §6.4). In addition, our weakly supervised method outperforms semi- and fully-supervised baselines with similar supervision cost. For the Pedestrian class with fewer annotations, our method even outperforms most existing fully supervised counterparts (§6.3), which further verifies its good generalizability.

Second, once trained, our detector can be applied as a 3D object annotation tool to assist the laborious labeling process. Current popular, supervised solutions eagerly consume all the training data to improve performance, while paying little attention to how to facilitate training data annotation. Our model design allows both automatic and active (semi-automatic) working modes. Our experiments (§6.5) show that, in the automatic mode, after directly applying our model to automatically re-annotate KITTI, re-trained PointPillars [7] and PointRCNN [16] can maintain more than $95 \%$ of their original performance. In the active setting, human annotators first provide center-click supervision on the BEV maps, which is used as privileged information to guide Stage-2 for final cuboid prediction. Under such a setting, re-trained PointPillars and PointRCNN reach above $97 \%$ of their original performance (with KITTI ground-truth annotations), which shows the high utility of our model.

The contributions in this work are summarized follows:

- An early attempt towards weakly supervised 3D object detection in the field of autonomous driving. Our model promises to offset the strong and costly supervision requirements of its fully supervised counterparts by exploiting weaker and fewer annotations (§3). Through our proposed easily-acquired center-click supervision on BEV maps as well as a few instance-wise 3D annotations, it learns a robust $3 \mathrm{D}$ object detector, which shows promising results on different object categories and has the potential to benefit from more weakly labeled training data.

- A novel network design allows accessing 3D object detection with low-cost labels. To make full use of above two kinds of supervision, our detector is elaborately designed as a two-stage architecture ( $\$ 4)$. Stage- 1 learns to generate cylinder-shaped vehicle candidates, to address the lack of height information in the BEV center-click annotations. Stage-2, which consumes a few instance-wise cuboid annotations (i.e., it uses partially labeled scenes), learns to refine the object proposals in a step-by-step manner. The cascade network design empowers Stage-2 with a higher learning capacity, and gives rise to more precise 3D object prediction by removing background noise under the guidance of the former output.

- An easily accessible annotator can label point clouds in alternative modes for different requirements. Once trained, the proposed 3D object detector can be adopted as an annotator, which allows both automatic and active modes (§6.5). While the automatic mode has the ability to label point cloud data by itself at a high speed $(0.1 \mathrm{~s}$ per instance), the active mode can achieve higher accuracy 
with minimum human click assistance. Our model shows high utility when acting as an annotation tool; its generated labels, when used to train fully supervised methods, yield similar supervision power to the manual labels.

This paper builds upon our conference paper [1] and extends it in various aspects. First, we improve our model with a cascaded network structure, for more efficient feature extraction and multi-step 3D prediction refinement ( $\$ 4.2)$. Second, to facilitate future efforts and better understand the advantages of our weakly supervised framework, we provide several semi-supervised baselines, derived from current top-leading fully supervised solutions (§6.2). Third, extensive experiments are conducted to demonstrate the effectiveness of our model, including exploring its robustness on different classes, and providing visual results (§6.2$\S 6.3)$. Forth, more ablation studies are designed for thorough examination (§6.4). Last but not least, we provide more discussions on the common failure cases and identify promising directions for future efforts $(\S 6.6)$.

\section{Related Work}

Learning Point Cloud Representations: Processing sparse, unordered point cloud data from LiDAR sensors is a fundamental problem in many 3D related areas. There are two main paradigms for this: voxelization or point based methods. The first type of methods [11], [12], [20], [21] voxelize point clouds into the volumetric grids and apply 2D/3D CNNs for prediction. Some of them [9], [14], [22], [23] further improve volumetric features with multi-view representations of point clouds. These methods are computationally efficient but suffer from information loss (due to quantitization of point clouds with coarse voxel resolution). The second type, point based methods [13], [16], [17], [24]-[26], which directly operate on raw point clouds and preserve the original information, have recently become popular.

3D Object Detection: A flurry of techniques have been explored for 3D object detection in driving scenarios, which can be broadly categorized into three classes. (1) $2 D$ image based methods focus on camera based solutions with monocular or stereo images, by exploring geometries between 3D and 2D bounding boxes [2], [4], [27], [28], or similarities between 3D objects and CAD models [3], [29]. Though efficient, they typically struggle against the inherent difficulty of directly estimating depth information from images. (2) $3 D$ point cloud based methods rely on depth sensors such as LiDAR. Some representative models project point clouds to $\mathrm{BEV}$ and use 2D CNNs [5], [9] to learn the point cloud features for 3D pose regression. Some others [17], [22] apply 3D CNNs over point cloud voxels to generate 3D boxes. They tend to capture local information, due to the limited receptive fields of CNN kernels. Thus sparse convolutions [15] with enlarged receptive fields are adopted later. To avoid losing information during voxelization, some efforts learn point-wise features directly from raw point clouds, using PointNet [13]-like structures [6], [7], [30]. (3) Fusion-based methods [13], [16], [25], [26], [31], [32] attempt to fuse information from different sensors, such as cameras and LiDAR. The basic idea is to leverage the complementary information of camera images and point clouds, i.e., the rich visual information of images and precise depth details of point clouds, to improve the detection accuracy. However, fusionbased methods typically run slowly due to the need of processing multi-modal inputs [15]. The additional requirement of time synchronization and calibration between camera and LiDAR also reduces their applicability and robustness [15].

Click Supervision: In 2D vision, click annotation schemes are widely used to reduce the burden of collecting segmentation/bounding box annotations at a large scale. Current efforts typically leverage center-click [33], [34], extremepoint [35], [36], or corrective-click [33] as supervision forms, and greatly benefit semantic segmentation [33], [37] and object detection [34]-[36] in 2D visual scenarios. However, in this work, we explore center clicks, located on BEV maps, as weak supervisory signals for 3D detection in driving scenes. Methods for Reducing Supervision in 3D Vision: Due to the significant difficulty and high cost of obtaining large quantities of labeled 3D data, research efforts in 3D vision have long addressed the need for annotation-efficient learning. A variety of techniques were developed to reduce the requirement for large quantities of 3D supervision. The most typical strategy is to utilize synthetic data to quickly build large-scale 3D datasets [38]-[41], which has benefited a lot of tasks, such as 3D flow estimation [42] and 3D human reconstruction [43]. Some attempts [44], [45] were made to learn indoor 3D detection through semi-supervised learning techniques, i.e., combine labeled data with unlabeled data. Some more recent efforts [46], [47] showed the great potential of unsupervised learning in $3 \mathrm{D}$ vision, where the model pretrained on unlabeled data can gain promising performance after supervised finetuning. Our work explores BEV center-clicks as a weak supervision for outdoor 3D detection. It will be also interesting to explore these annotation-reducing strategies in 3D driving scenes.

3D Object Annotation: Very few attempts have been made to scale up 3D object annotation pipelines [48], [49]. Specifically, [48] lets an annotator place 2D seeds from which to infer 3D segments and centroid parameters, based on a fully supervised deep learning framework. In [49], an automatic 3D annotation model is proposed, achieved by a differentiable template matching strategy with curriculum learning. In addition to its different annotation paradigms, model designs and levels of human intervention, our model is also unique in its weakly supervised learning strategy and dual-work mode, and achieves stronger performance.

\section{Data annotation Strategy for Our BeV Center-Click Supervision}

Before elaborating on our weakly supervised 3D detector $(\S 4)$, we first detail how to get our weak BEV annotations.

Traditional Precise But Laborious Labeling Strategy: Current popular 3D object detectors are fully supervised deep learning models, requiring a large amount of precisely annotated data. However, creating a high-quality, largescale 3D object detection dataset is more complex than creating, for example, a 2D object detection dataset. For precise labeling [50], [51], annotators usually first navigate the 3D scene to find an object with the help of visual content from the camera image (Fig. 3(a)). Then, an initial rough cuboid and orientation arrow (Fig. 3(b)) are placed. Finally, the optimal annotation (Fig. 3(c)) is obtained by gradually 

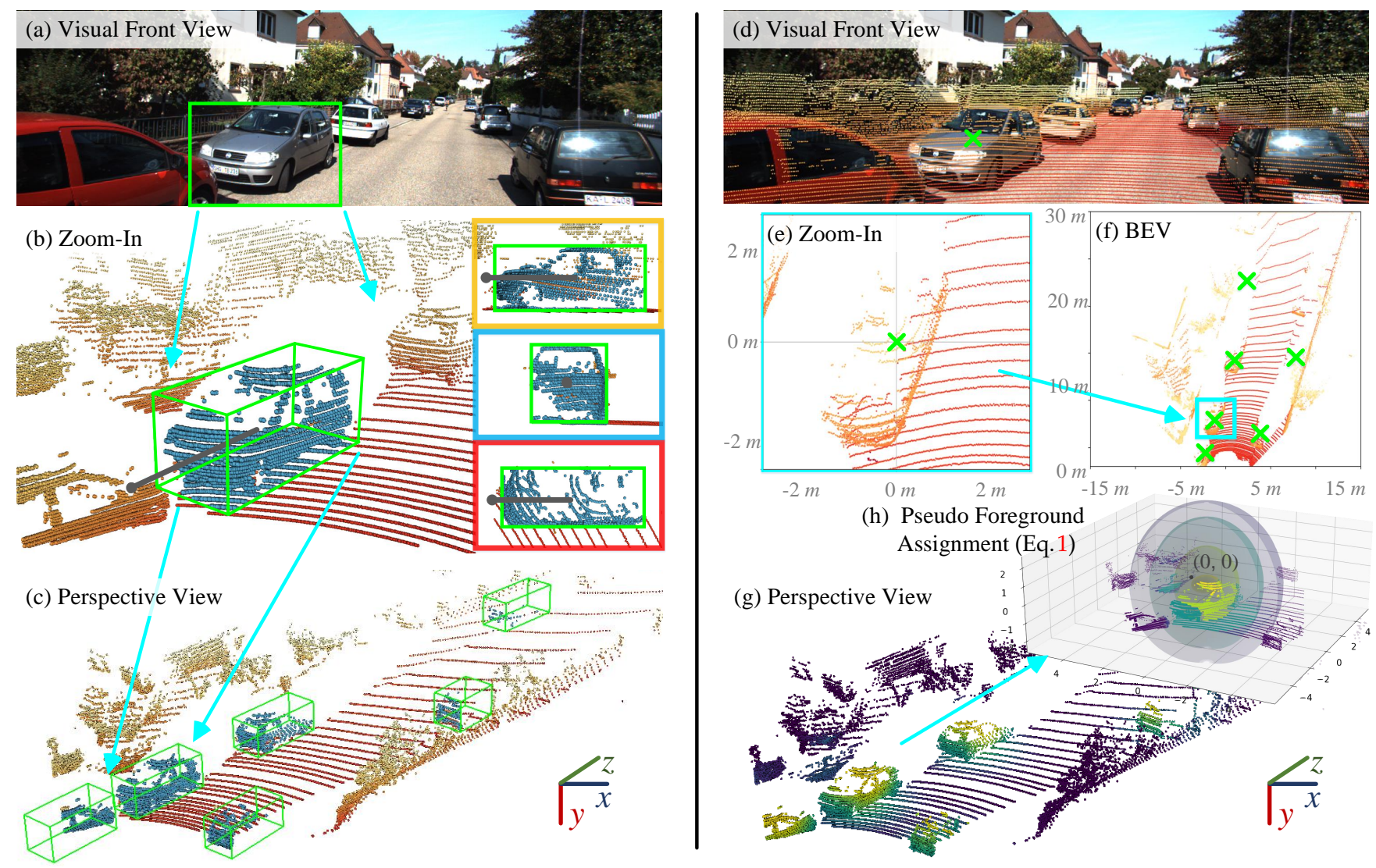

(h) Pseudo Foreground Assignment (Eq.1)

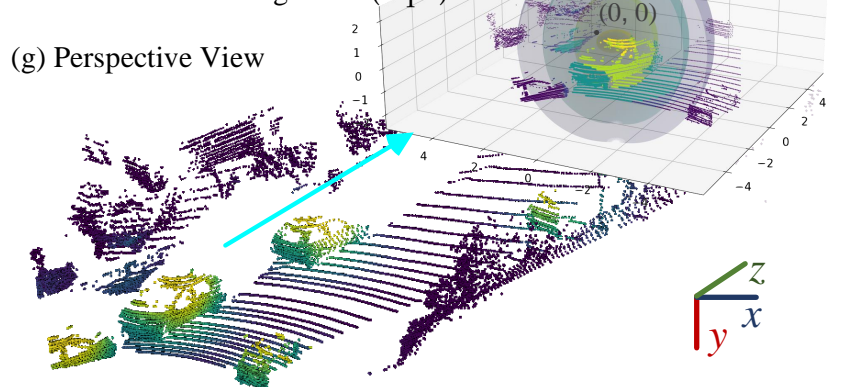

Fig. 3. (a-c) Precise annotations require extensive labeling efforts (§3). (d-f) Our weak supervision is simply obtained by center-clicks (denoted by *) on BEV maps (§3). (g-h) Our pseudo ground-truths for fore-/background segmentation (yellower indicates higher foreground score; $\S 4.1$ ).

adjusting the 2D boxes projected in orthographic views. As can be seen, although this labeling procedure generates high-quality annotations, it contains several subtasks with gradual corrections and $2 \mathrm{D}-3 \mathrm{D}$ view switches. It is thus quite laborious and expensive.

Our Weak but Fast Annotation Scheme: Our model is learned from a small set of weakly annotated BEV maps, combined with a few precisely labeled 3D object instances. The weakly annotated data only contains object centerannotated BEV maps, which can be easily obtained. Specifically, human annotators first roughly click a target on the camera front-view map (Fig. 3(d)). Then, the BEV map is zoomed in and the region around the initial click is presented for a more accurate center-click (Fig. 3(e)). Since our annotation procedure does not refer to any $3 \mathrm{D}$ view (only need visual front view and BEV map), it is easy and fast; most annotations can be finished by only two clicks. The collected supervision is weak, as only the object centers over the $(x, z)$-plane are labeled, without height information in the $y$-axis or cuboid size.

Annotation Speed: We relabeled KITTI train set [19], which has 3,712 driving scenes with more than $15 \mathrm{~K}$ vehicle instances. This took about 11 hours, i.e., $2.5 \mathrm{~s}$ per instance. As KITTI does not report the original annotation time, we refer to other published statistics [51], [52], which suggest around $114 \mathrm{~s}$ per instance in a fully manual manner [52] or $30 \mathrm{~s}$ with extra assistance of a 3D object detector [51]. Thus our click supervision provides a $15 \sim 45 \times$ reduction in the time required for traditional precise annotations.

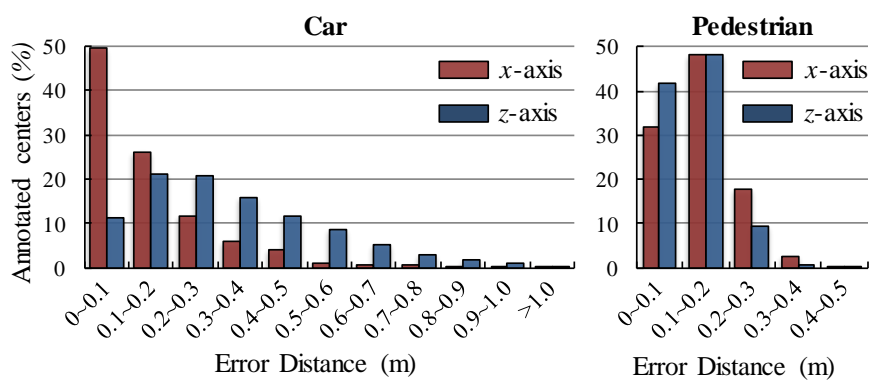

Fig. 4. Error distributions of our weak BEV annotations.

Annotation Quality: To assess our annotation quality, we report the average distance between our annotated centers and KITTI ground-truths on BEV maps, in Fig. 4. For each annotated center, the corresponding error is computed as the distance to its nearest ground-truth center. For Car class, the average errors on $x$ - and $z$-axes are about $0.25 \mathrm{~m}$ and $0.75 \mathrm{~m}$, respectively, bringing out the limitation of LiDAR sensors in capturing the side of an object than its back.

\section{Methodology}

Our object detector takes raw point clouds as input and outputs oriented 3D boxes. It has two stages. Stage- 1 involves roughly extracting foreground points and generating cylindrical 3D candidates, learning from our BEV centerclick supervision (§4.1, Fig. 5(a-b)). Then, Stage-2 refines the 


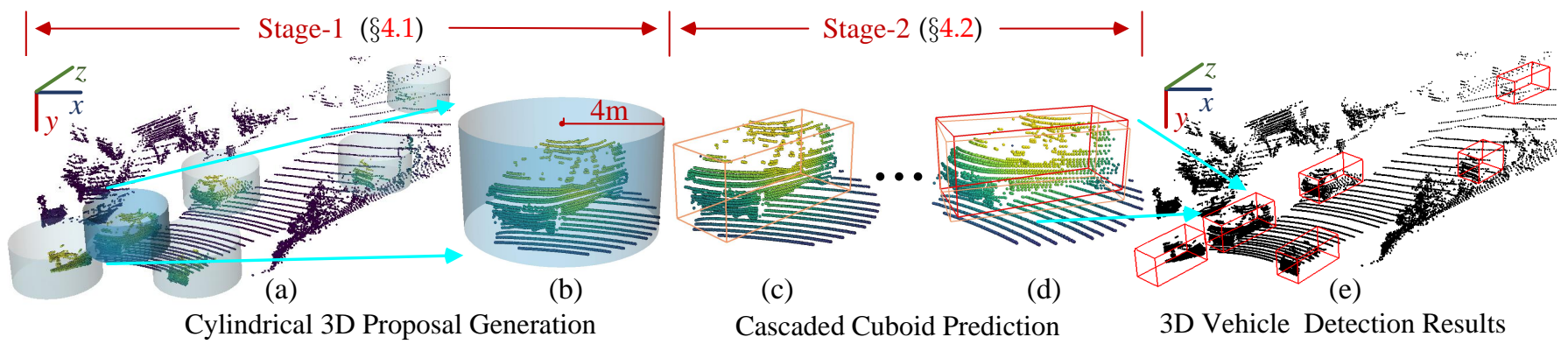

Fig. 5. Our 3D object detection pipeline ( $\$ 4)$. (a-b) Cylindrical 3D proposal generation results from Stage-1 ( $\$ 4.1)$. Yellower colors correspond to higher foreground probabilities. (c-d) Cascaded cuboid prediction in Stage-2 (§4.2). (e) Our final 3D object results.

object proposals and estimates the confidence of the output 3D bounding boxes in coarse-to-fine manner, learning from a few, well-annotated vehicle training instances $(\S 4.2$, Fig. 5(c-d)). Below we will focus on the Car class. However, as evidenced in our experiments (§6.3), our model can also easily be applied to other classes, such as Pedestrian.

\subsection{Stage-1: Learning to Generate Cylindrical 3D Pro- posals from BEV Center-Click Annotations}

There are two goals in our first stage: 1) to generate an initial foreground point segmentation; and 2) to produce a set of cylindrical 3D object proposals. The fore-/background separation is helpful for the proposal generation and provides useful information for Stage-2. As only the horizontal centers of objects are labeled on the BEV maps, our proposals are cylinder-shaped, instead of being 3D bounding boxes.

Pseudo Ground-Truth Generation. Since the annotations in the BEV maps are weak, proper modifications should be made to produce pseudo, yet stronger supervisory signals. Specifically, for a labeled vehicle center point $o \in \mathcal{O}$, its horizontal location $\left(x_{o}, z_{o}\right)$ in the LiDAR coordinate system can be inferred according to the projection from the BEV to the point cloud. We set its height $y_{o}$ (over the $y$-axis) to the LiDAR sensor's height (the height of the ego-vehicle), i.e., $y_{o}=0$. The rationale behind such a setting will be detailed later. Then, for each unlabeled point $p$, its pseudo foreground value $f^{p} \in[0,1]$ is defined as:

$$
\begin{aligned}
f^{p} & =\max _{o \in \mathcal{O}}(\iota(p, o)), \\
\text { where } \iota(p, o) & = \begin{cases}1 & \text { if } d(p, o) \leq 0.7, \\
\frac{1}{\kappa} \mathcal{N}\left(d(p, o) ; \mu, \sigma^{2}\right) & \text { if } d(p, o)>0.7 .\end{cases}
\end{aligned}
$$

Here, $\mathcal{N}\left(x ; \mu, \sigma^{2}\right)$ is a $1 \mathrm{D}$ Gaussian distribution with mean $\mu=0.7$ and variance $\sigma^{2}=1.5$, and $\kappa=\mathcal{N}\left(0.7 ; \mu, \sigma^{2}\right)$ is a normalization factor. Further, $d(p, o)$ is a distance function: $d(p, o)=\left[\left(x_{p}-x_{o}\right)^{2}+\lambda\left(y_{p}-y_{o}\right)^{2}+\left(z_{p}-z_{o}\right)^{2}\right]^{\frac{1}{2}}$, where $\left(x_{p}, y_{p}, z_{p}\right)$ are the $3 \mathrm{D}$ coordinate of $p$. The coefficient $\lambda$ $(=0.5)$ is used due to the large uncertainty over the $y$ axis. As the BEV annotations do not provide height information, if a large coefficient $\lambda$ is adopted, many foreground points will be excluded (i.e., blue points in Fig. 6), causing performance decrease. Basically, the foreground probability assignment function $\iota(p, o)$ gives high confidence $(=1)$ for those points close to $o$ (i.e., $d(p, o) \leq 0.7)$, and attenuates the confidence for distant ones (i.e., $d(p, o)>0.7)$, following the Gaussian distribution $\mathcal{N}$. As most vehicles are seemingly

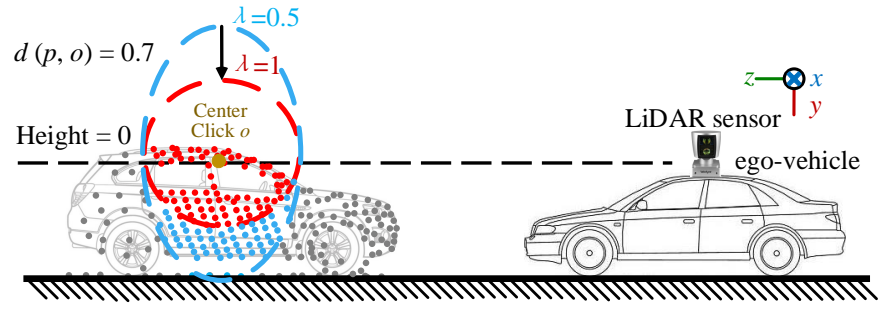

Fig. 6. Illustration of our pseudo ground-truth generation strategy (Eq. 1).

of a similar height, for each labeled center $o$, we roughly set its height $(=0)$ as the altitude of the Lidar sensor, which is placed on the ego-vehicle (i.e., right car in Fig. 6). For the points that are close to $o$ and at lower altitudes than the LiDAR sensor, most of them are object points and will gain high foreground values. For the points above the egovehicle, there may exist some background points which are assigned high foreground scores, but the amounts are trivial. This is because, at the similar altitudes to the LiDAR sensor, background points are very sparse and typically far away from the vehicle centers in the $(x, z)$-plane (see Fig. 3(h)). Note that, if we set the heights of labeled center points a smaller value, many background points on the ground will gain high foreground scores. Plane detection [4] can be also used here for more accurate height estimation, but in practice we find our strategy is already good enough.

Point Cloud Feature Extraction. For point cloud feature extraction, an encoder-decoder network like [25] is adopted. In the encoder, a stack of set-abstraction layers with multiscale grouping is applied to extract raw point cloud features. Then, the decoder, which consists of several feature propagation layers, is added to learn more discriminative pointwise features. Finally, two branches are placed over the feature extraction backbone for foreground point segmentation and object $(x, z)$-center regression, respectively.

Foreground Point Segmentation. With the point-wise features extracted from the backbone network and the pseudo ground-truth $f^{p}$ generated by Eq. 1, we use a foreground segmentation branch for estimating the foreground probability $\tilde{f}^{p} \in[0,1]$ of each point $p$. The learning is achieved by minimizing the following loss:

$$
\begin{aligned}
\mathcal{L}_{\text {seg }} & =-\alpha\left(1-\hat{f}^{p}\right)^{\gamma} \log \left(\hat{f}^{p}\right), \\
\hat{f}^{p} & =\tilde{f}_{p} \cdot f^{p}+\left(1-\tilde{f}^{p}\right) \cdot\left(1-f^{p}\right) .
\end{aligned}
$$

This is a soft version of the focal loss [53], which is used to 
address the foreground-background imbalance in outdoor scenes. As in [53], we set $\alpha=0.25$ and $\gamma=2$.

Object $(x, z)$-Center Prediction. The other branch is for object $(x, z)$-center regression, as the weakly annotated BEV maps only contain horizontal information. As in [16], a binbased classification strategy is adopted. For each labeled object center $o \in \mathcal{O}$, we set the points within a $4 \mathrm{~m}$ radius as support points (whose foreground probabilities $f^{p} \geq 0.1$ ). These support points are used to estimate the horizontal coordinates of $o$. For each support point $p$, its surrounding area $\left(L \times L \mathrm{~m}^{2}\right)$ over the $(x, z)$-plane is the searching space for $o$, which is split into a series of discrete bins. Concretely, for the $x$ - and $z$-axes, the search range $L(=8 \mathrm{~m})$ is divided into 10 bins of uniform length $\delta(=0.8 \mathrm{~m})$. Thus for a support point $p$ and the corresponding center $o$, the target bin assignments $\left(b_{x}, b_{z}\right)$ along the $x$ - and $z$-axes can be formulated as:

$$
b_{x}=\left\lfloor\frac{x_{p}-x_{o}+\frac{L}{2}}{\delta}\right\rfloor, \quad b_{z}=\left\lfloor\frac{z_{p}-z_{o}+\frac{L}{2}}{\delta}\right\rfloor .
$$

Residual $\left(r_{x}, r_{z}\right)$ is computed for further location refinement within each assigned bin:

$$
r_{u \in\{x, z\}}=\frac{1}{\varepsilon}\left(u_{p}-u_{o}+\frac{L}{2}-\left(b_{u} \cdot \delta+\frac{\delta}{2}\right)\right),
$$

where $\varepsilon=\frac{\delta}{2}$ is used for normalization. For a support point $p$, the center localization loss $\mathcal{L}_{\text {bin }}$ is designed as:

$$
\mathcal{L}_{\text {bin }}=\sum_{u \in\{x, z\}} \mathcal{L}_{\mathrm{cls}}\left(\tilde{b}_{u}, b_{u}\right)+\mathcal{L}_{\text {reg }}\left(\tilde{r}_{u}, r_{u}\right)
$$

where $\tilde{b}_{x}$ and $\tilde{r}_{x}$ (resp. $\tilde{b}_{z}$ and $\tilde{r}_{z}$ ) are predicted bin assignments and residuals, and $b_{x}$ and $r_{x}$ (resp. $b_{z}$ and $r_{z}$ ) are the targets, over the $x$-axis (resp. $z$-axis). $\mathcal{L}_{\text {cls }}$ is a cross-entropy loss for bin classification, and $\mathcal{L}_{\text {reg }}$ refers to the $\ell 1$ loss for residual regression w.r.t the target bins. $\mathcal{L}_{\text {cls }}$ and $\mathcal{L}_{\text {reg }}$ are computed over the $x$ - and $z$-axes, separately.

Cylindrical 3D Proposal Generation. During inference, the segmentation branch estimates the foreground probability of each point. Then, we only preserve the points whose foreground scores are larger than 0.1. We choose the bin center with the highest predicted confidence and add the predicted residual to obtain the refined parameters. As we only have horizontal coordinates of the centers, we cannot directly generate 3D bounding box proposals. Instead, for each center, we generate a cylindrical (vertical columnshaped) proposal with a $4 \mathrm{~m}$ radius over the $(x, z)$-plane and unlimited length along the $y$-axis (Fig. $5(\mathrm{a}, \mathrm{b})$ ).

Objectness-Score based Non-Maximum Suppression. To eliminate redundant proposals, we propose an ObjectnessScore based non-maximum suppression (OB-NMS) strategy. The main idea is that it is easier to predict centers from center-close points than far ones, and center-close points gain high foreground scores under our pseudo ground-truth generation strategy. Thus, for a predicted center, we use the foreground probability of its sourced (support) point, as its confidence score. This means we assume that a point with a higher foreground score is more center-close and tends to make a more confident center prediction. Then we rank all the predicted centers according to their confidence, from large to small. For each center, if its distance to any other pre-selected center is larger than $0.3 \mathrm{~m}$ on the $(x, z)$-plane, its proposal will be preserved; otherwise it is removed.
4.2 Stage-2: Learning to Refine Proposals in a Coarseto-Fine Manner from a Few Well-Labeled Instances

Stage-2 involves estimating cuboids from proposals and recognizing false estimates. We achieve this by learning a cascaded proposal refinement model from a limited number of precise 3D box annotations. Three considerations motivate our model design. First, the proposal refinement is performed instance-wise, driving us to consume instancelevel annotations, instead of exhaustively annotated scenes. Second, our initial cylindrical proposals, though rough, contain rich and useful information, which facilitates cuboid prediction especially when training data is limited. Third, inspired by the success of cascaded 2D object detectors [54] [56], we perform coarse-to-fine proposal refinement, yielding promising results with higher learning capacity.

Overall Pipeline. Our method carries out progressive refinement of cuboid predictions over multiple steps. At each step, a proposal refinement network takes cylindrical proposals or cuboids from the previous step as inputs, and outputs more accurate cuboid estimations (Fig. 5(b-d)). The refinement networks in different steps have the same architecture, but do not share weights. In the final step, we let the refinement network predict both cuboids and confidence. As such, all the 3D parameters are regressed in a cascaded and gradually improved manner, yielding a compact yet powerful proposal refinement framework.

Cascaded Proposal Refinement Network. The proposal refinement network stacks several set abstraction layers, intermediated with a single-scale grouping operation, to collect contextual and pooled point features as the proposal/cuboid representations [25]. Then, a multilayer perceptron based branch is appended for cuboid prediction. Let us denote the ground-truth of an input proposal/cuboid as $(x, y, z, h, w, l, \theta)$, where $(x, y, z)$ are the object-center coordinates, $(h, w, l)$ object size, and $\theta$ orientation on the BEV. For each proposal refinement network, a bin-based regression loss $\mathcal{L}_{\text {bin }}$ is applied for estimating $\theta$ (with 12 bins in total), and a smooth $\ell 1$ loss $\mathcal{L}_{\text {reg }}$ for other parameters:

$$
\mathcal{L}_{\text {ref }}=\mathcal{L}_{\text {bin }}(\tilde{\theta}, \theta)+\Sigma_{u \in\{x, y, z, h, w, l\}} \mathcal{L}_{\text {reg }}(\tilde{u}, u) .
$$

Here $(\tilde{x}, \tilde{y}, \tilde{z}, \tilde{h}, \tilde{w}, \tilde{l}, \tilde{\theta})$ are the estimated cuboid parameters. IoU-Based Confidence Estimation. To predict the cuboid's confidence, an extra confidence estimation branch is added into the last proposal refinement network. By feeding the cuboid's features into the confidence estimation head, the $\ell 1$ loss is applied for supervising the IOU-based confidence prediction [28], [57]:

$$
\mathcal{L}_{\text {con }}=\mathcal{L}_{\text {reg }}\left(\tilde{C}_{\text {IoU }}, C_{\text {IoU }}\right),
$$

where the targeted confidence score $C_{\mathrm{IoU}}$ is computed as the IoU between the latest output cuboid and ground-truth.

Learning Cascaded Inference. For the cascaded inference, current step gains assistance from the prior step, by gradually removing background noise and outputting more accurate cuboid estimations. Specifically, in the first proposal refinement step, for each ground-truth 3D bounding box, cylindrical proposals whose center-distances (on the $(x, z)$ plane) are less than $1.4 \mathrm{~m}$ away are selected as the training samples. Then, the cuboids estimated from those cylindrical proposals are further used as the training samples for the 
ground-truth in the next proposal refinement step. After several refinement steps, the outputs from the last proposal refinement network are our final 3D object estimates. In this way, our Stage-2 module learns to perform coarse-to-fine proposal refinement, where the current step improves the outputs of the previous step. In our implementation, we set the inference steps as 3 (see related experiments in $\S 6.4$ ).

\section{Implementation Details}

Detailed Network Architecture. In Stage-1 (§4.1), to align the network input, $16 \mathrm{~K}$ points are sampled from each pointcloud scene, following [16]. Four set-abstraction layers of multiple scales are stacked to sample the points into groups with sizes $(4096,1024,256,64)$. Four feature propagation layers are then used to obtain $16 \mathrm{~K}$ point-wise features (each of which is a $512 \mathrm{D}$ vector), as the input for both the segmentation and center prediction branches. The segmentation branch has two fully connected (FC) layers with 128 and 1 neuron(s), respectively. The $(x, z)$-center prediction branch has two FC layers with 128 and 40 neurons, respectively.

In Stage-2 (§4.2), 512 points are sampled from each cylindrical proposal/cuboid, and each point is associated with a 5D feature vector, i.e., a concatenation of 3D point coordinates, 1D laser reflection intensity, and foreground score. Each step repeatedly canonizes the input point cloud and progressively removing background noise. Specifically, before feeding each proposal/cuboid into the refinement network, the coordinates of points are canonicalized to guarantee their translation and rotation invariance [6], [16]. The corresponding ground-truth is modified accordingly. In the canonical coordinate system, the origin is located at the center of the cuboid, the local $(x, z)$-plane of the cuboid is parallel to the ground plane with the $x$-axis pointing towards the head direction of the cuboid, and the local $y$ axis maintains unchanged [16]. Note that, for the cylindrical proposals, only a translation transformation is performed over the $(x, z)$-plane, i.e., the $(x, z)$-center of the proposal is set as $(0,0)$. For each generated cuboid, the points within a $0.3 \mathrm{~m}$ radius are cropped to include more context. For the proposal refinement network in each step, four setabstraction layers with single-scale grouping are used to sample the input points into groups with sizes $(256,128$, 32,1 ), outputting a $512 \mathrm{D}$ feature. Then two FC layers (with 256 and 30 neurons respectively) are stacked for cuboid prediction. For the last proposal refinement network, an extra IOU scoring branch is appended, which contains two FC layers with 256 and 1 neuron(s), respectively.

Data Augmentation. During training, we adopt several data augmentation techniques to avoid overfitting and improve data diversity. In Stage-1, left-right flipping, scaling from $[0.95,1.05]$, and rotation from $\left[-10^{\circ}, 10^{\circ}\right]$ are randomly applied for each scene. To further diversify training scenarios, we randomly sample a few annotated vehicle centers with surrounding points within a cylinder with a $4 \mathrm{~m}$ radius, and insert them into the current sample. Furthermore, to increase the robustness to distant vehicles, which typically contain very few points, we randomly drop the points within the cylindrical space (with a $4 \mathrm{~m}$ radius) of labeled centers. In Stage-2, for each proposal, we randomly conduct left-right flipping, scaling from [0.8, 1.2], and rotation from $\left[-90^{\circ}, 90^{\circ}\right]$. We shift each proposal by small translations, following a Gaussian distribution with mean 0 and variance 0.1 , for the $x_{-}, y^{-}$, and $z$-axes each individually. We also randomly change the foreground label of the points. In addition, to address large occlusions, we randomly omit sections of a proposal $(1 / 4-3 / 4$ of the area in the BEV). Finally, for each proposal, we randomly remove the inside points (at least 32 points remain).

Configuration and Reproducibility. Our model is implemented in PyTorch and trained on eight Nvidia Tesla V100 GPUs with 32GB memory per card. Our implementations and labeled data (\$3) are available at https://github.com/ hlesmqh/WS3D, to provide full details of our algorithms.

\section{EXPERIMENTS}

\subsection{Experimental Setup}

Dataset. We conduct experiments on KITTI [19], a standard benchmark in the field of 3D object detection. This dataset contains 7,481 images for train/val and 7,518 images for testing, with the aim of evaluating object detection for vehicles, pedestrians and cyclists from images or LiDAR point clouds. The train/val set has annotated groundtruths with $2 \mathrm{D}$ bounding boxes in the image plane and 3D bounding boxes for scan data. Following [9], [17], we split train/val set into training and validation sub-sets, resulting in 3,712 data samples for training and 3,769 for validation. Such a split ensures that the images from the same sequence do not exist in both train and val sets.

Evaluation Protocol. We train our detector on a weakly labeled subset of train set (detailed later), while val set is used for evaluation only. Detection outcomes are evaluated under the three standard regimes: easy, moderate, hard, defined according to occlusion and truncation levels of objects. Evaluation Metric. As conventional [9], average precisions (APs) for BEV and 3D boxes are reported. Unless specified, the performances on the Car and Pedestrian classes are evaluated with IoU thresholds of 0.7 and 0.5 , respectively.

\subsection{Performance on 3D Car Detection}

We first conduct experiments on the Car class of KITTI, as is common [7], [16], [17], [26].

- Data Preparation. The 3,712 training scenes in KITTI contain 15,654 vehicle instances in total. The first 500 scenes with our weakly annotated BEV maps are used for training our Stage- 1 model, while $25 \%$ of the vehicle instances $(=534)$ in the 500 scenes are associated with precise 3D annotations and used for training our Stage-2 model $^{1}$. We make use of this weak and limited training data to better illustrate the advantage of our model. This also allows us to investigate the performance when using our model as an annotation tool (see $\S 6.5$ ).

- Training. We use the Adam optimizer with an initial learning rate of 0.002 and weight decay of 0.0001 . In Stage1 (§4.1), we train the network for $8 \mathrm{~K}$ iterations with a

1. As the training data of KITTI have been already randomly shuffled, we directly choose the first 500 scenes for training. This makes future efforts easy to follow the experimental protocol. The precisely annotated instances in the first 500 scenes are randomly selected and the list can be found at our website (https://github.com/hlesmqh/WS3D). 
TABLE 1

Evaluation results ( $\S 6.2$ ) on val set of KITTI 3D and BEV object detection benchmark (Car). AP with loU 0.7 threshold is reported.

\begin{tabular}{|c|c|c|c|c|c|c|c|c|}
\hline \multirow{2}{*}{ Learning Paradigm } & \multirow{2}{*}{ Detector } & \multirow{2}{*}{ Modality } & \multicolumn{3}{|c|}{ BEV@0.7 } & \multicolumn{3}{|c|}{ 3D Box@0.7 } \\
\hline & & & Easy & Moderate & Hard & Easy & Moderate & Hard \\
\hline \multicolumn{9}{|c|}{ Trained with the whole KITTI train set: all 3, 712 precisely labeled scenes with 15,654 vehicle instances } \\
\hline \multirow{11}{*}{ Fully supervised } & MV3D [9] & LiDAR+Mono & 86.55 & 78.10 & 76.67 & 71.29 & 62.68 & 56.56 \\
\hline & F-PointNet [26] & LiDAR+Mono & 88.16 & 84.02 & 76.44 & 83.76 & 70.92 & 63.65 \\
\hline & MMF [32] & LiDAR+Mono & 96.66 & 88.16 & 79.60 & 87.90 & 77.86 & 75.57 \\
\hline & VeloFCN [14] & LiDAR & 40.14 & 32.08 & 30.47 & 15.20 & 13.66 & 15.98 \\
\hline & PIXOR [5] & LiDAR & 86.79 & 80.75 & 76.60 & - & - & - \\
\hline & VoxelNet [17] & LiDAR & 89.60 & 84.81 & 78.57 & 81.97 & 65.46 & 62.85 \\
\hline & SECOND [15] & LiDAR & 89.96 & 87.07 & 79.66 & 87.43 & 76.48 & 69.10 \\
\hline & PointRCNN [16] & LiDAR & - & - & - & 88.45 & 77.67 & 76.30 \\
\hline & PointPillars [7] & LiDAR & 89.64 & 86.46 & 84.22 & 85.31 & 76.07 & 69.76 \\
\hline & Fast PointR-CNN [6] & LiDAR & 90.12 & 88.10 & 86.24 & 89.12 & 79.00 & 77.48 \\
\hline & STD [18] & LiDAR & 90.50 & 88.50 & 88.10 & 89.70 & 79.80 & 79.30 \\
\hline \multicolumn{9}{|c|}{ Trained with a part of KITTI train set: first 500 precisely labeled scenes with 2,176 vehicle instances } \\
\hline \multirow{2}{*}{ Fully supervised } & PointRCNN [16] & LiDAR & 87.21 & 77.10 & 76.63 & 79.88 & 65.50 & 64.93 \\
\hline & PointPillars [7] & LiDAR & 86.27 & 77.13 & 75.91 & 72.36 & 60.75 & 55.88 \\
\hline \multicolumn{9}{|c|}{ Trained with a part of KITTI train set: first 125 precisely labeled scenes with 550 vehicle instances } \\
\hline \multirow{2}{*}{ Fully supervised } & PointRCNN [16] & LiDAR & 85.09 & 74.35 & 67.68 & 67.54 & 54.91 & 51.96 \\
\hline & PointPillars [7] & LiDAR & 85.76 & 75.30 & 73.29 & 65.51 & 51.45 & 45.53 \\
\hline \multicolumn{9}{|c|}{ Trained with a part of KITTI train set: first 500 precisely labeled scenes with 2, 176 vehicle instances + rest scenes without label } \\
\hline \multirow{2}{*}{ Semi supervised } & PointRCNN [16] & LiDAR & 87.50 & 81.32 & 81.45 & 80.12 & 67.43 & 68.30 \\
\hline & PointPillars [7] & LiDAR & 88.85 & 79.65 & 78.59 & 74.36 & 61.05 & 56.69 \\
\hline \multicolumn{9}{|c|}{ Trained with a part of KITTI train set: first 125 precisely labeled scenes with 550 vehicle instances + rest scenes without label } \\
\hline \multirow{2}{*}{ Semi supervised } & PointRCNN [16] & LiDAR & 86.44 & 78.21 & 71.85 & 68.32 & 55.11 & 55.36 \\
\hline & PointPillars [7] & LiDAR & 86.83 & 75.74 & 74.32 & 66.65 & 51.52 & 46.72 \\
\hline \multicolumn{9}{|c|}{ Trained with a part of KITTI train set: first 500 weakly labeled scenes with 534 precisely annotated vehicle instances } \\
\hline Weakly supervised & Ours & LiDAR & 88.95 & 85.83 & 85.03 & 85.04 & 75.94 & 74.38 \\
\hline
\end{tabular}

batch-size of 25. In Stage-2 (§4.2), there are 40K training iterations in total, with a batch-size of 800 .

- Inference. After applying OS-NMS for the cylindrical proposals generated in Stage-1 ( $\$ 4.1)$, we feed the remaining ones to Stage-2 (\$4.2) and obtain final 3D predictions. We then use an oriented NMS with a BEV IoU threshold of 0.3 to reduce redundancy. Our model runs at about $0.2 \mathrm{~s}$ per scene, which is on par with MV3D [9] (0.36 s), VoxelNet [17] (0.23 s) and F-PointNet[26] (0.17 s).

Quantitative Results on KITTI val Set. In Table 1, we compare our method with several leading algorithms, including three multi-modal models [9], [26], [32] and eight LiDAR based models [5]-[7], [14]-[18]. The competitors all use the fully labeled training data (i.e., 3,712 preciselylabeled scenes with 15,654 vehicle instances). However, though using far less, weakly labeled data, our method still yields comparable performance.

As there is no other weakly supervised baseline, we re-train two outstanding detectors, PointRCNN [16] and PointPillars [7], under two relatively comparable supervision settings, i.e., using (i) 500 precisely labeled scenes (containing 2,176 well-annotated vehicle instances); and (ii) 125 precisely labeled scenes (containing 550 well-annotated instances). This helps to further assess the effectiveness of our method. For our model, we use 500 scenes with centerclick labels and 534 precisely-annotated instances. Table 1 shows that our method significantly outperforms re-trained PointRCNN and PointPillars, using both the same amount of well-annotated scenes (setting (i)) and the same number of well-annotated instances (setting (ii)).
To further demonstrate our advantages and benefit future efforts, we provide several semi-supervised baselines. Specifically, PointRCNN (or PointPillars) is first trained on the first 500 or 125 precisely labeled scenes. Then, we use the trained PointRCNN (or PointPillars) to generate pseudo ground-truths for the remaining unlabeled data, and retrain PointRCNN (or PointPillars) over the whole train set of KITTI. Later, pseudo label generation and retraining are iteratively conducted until convergence (performance change on KITTI val set is less than $1 \%$ after retraining, measured by 3D AP with 0.7 IoU threshold). From Table 1 we can easily observe that, for both PointRCNN and PointPillars, the improvements with extra unlabeled data are marginal. This further verifies the superiority of our weakly supervised 3D object detection network design.

Qualitative Results on KITTI val Set. Fig.7 depicts visual results for a few representative scenes from KITTI val set. Our model is able to produce high-quality $3 \mathrm{D}$ detections of vehicles that are near the ego-vehicle and have dense point clouds (subfigures 2, 4 and 6). In addition, the model can give precise predictions for highly-occluded or far-away vehicles (subfigures 1, 3 and 5).

Quantitative Results on KITTI test Set. We also evaluate our algorithm on KITTI test set, by submitting our results to the official evaluation server. As shown in Table2, though current top-performing methods use much stronger supervision, our method still obtains competitive performance against some of them, such as PIXOR [5] and VoxelNet [17]. However, we also observe that there is still room for improvement between weakly and fully supervised methods. 
TABLE 2

Evaluation results ( $\S 6.2)$ on test set of KITTI 3D and BEV object detection benchmark (Car). AP with loU 0.7 threshold is reported.

\begin{tabular}{|c|c|c|c|c|c|c|c|c|}
\hline \multirow{2}{*}{ Learning Paradigm } & \multirow{2}{*}{ Detector } & \multirow{2}{*}{ Modality } & \multicolumn{3}{|c|}{$\overline{\text { BEV@0.7 }}$} & \multicolumn{3}{|c|}{ 3D Box@0.7 } \\
\hline & & & Easy & Moderate & Hard & Easy & Moderate & Hard \\
\hline \multicolumn{9}{|c|}{ Trained with the whole KITTI train set: all 3,712 precisely annotated scenes with 15,654 vehicle instances } \\
\hline \multirow{10}{*}{ Fully supervised } & MV3D [9] & LiDAR+Mono & 86.02 & 76.90 & 68.49 & 71.09 & 62.35 & 55.12 \\
\hline & F-PointNet [26] & LiDAR+Mono & 88.70 & 84.00 & 75.33 & 81.20 & 70.39 & 62.19 \\
\hline & MMF [32] & LiDAR+Mono & 89.49 & 87.47 & 79.10 & 86.81 & 76.75 & 68.41 \\
\hline & PIXOR [5] & LiDAR & 87.25 & 81.92 & 76.01 & - & - & - \\
\hline & VoxelNet [17] & LiDAR & 89.35 & 79.26 & 77.39 & 77.47 & 65.11 & 57.73 \\
\hline & SECOND [15] & LiDAR & 88.07 & 79.37 & 77.95 & 83.13 & 73.66 & 66.20 \\
\hline & PointRCNN [16] & LiDAR & 89.47 & 85.68 & 79.10 & 85.94 & 75.76 & 68.32 \\
\hline & PointPillars [7] & LiDAR & 88.35 & 86.10 & 79.83 & 79.05 & 74.99 & 68.30 \\
\hline & Fast PointR-CNN [6] & LiDAR & 88.03 & 86.10 & 78.17 & 84.28 & 75.73 & 67.39 \\
\hline & STD [18] & LiDAR & 94.74 & 89.19 & 86.42 & 87.95 & 79.71 & 75.09 \\
\hline \multicolumn{9}{|c|}{ Trained with a part of KITTI train set: first 500 weakly labeled scenes +534 precisely annotated vehicle instances } \\
\hline Weakly supervised & Ours & LiDAR & 90.96 & 84.93 & 77.96 & 80.99 & 70.59 & 64.23 \\
\hline
\end{tabular}
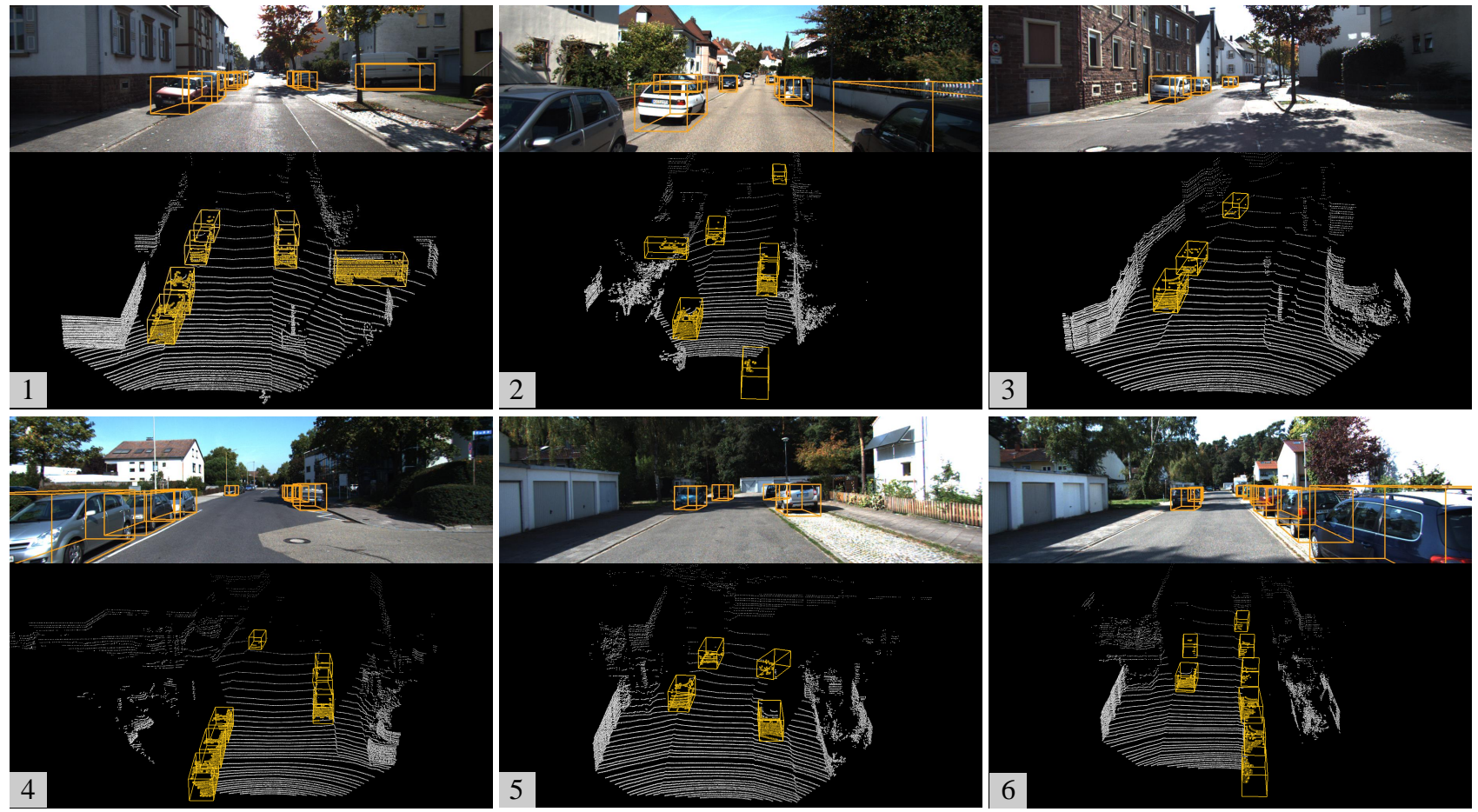

Fig. 7. Qualitative results of 3D object detection (Car) on KITTI val set (§6.2). (Detected 3D bounding boxes are shown in yellow; images are used only for visualization. Zoom-in for details. These notes are the same for the other figures.)

\subsection{Performance on Other Classes (Pedestrian)}

To further demonstrate the efficacy and generalizability of our weakly supervised 3D object detection framework, we conduct experiments on the Pedestrian class of KITTI. Note that acceptable predicted 3D boxes should have IoUs higher than 0.5 with the ground-truths.

- Data Preparation. For the 3, 712 training scenes in KITTI, there are only 951 containing Pedestrian labels. Considering the small amount of training samples, we use the weakly annotated BEV maps of the 951 scenes to train our Stage-1 model. We randomly choose 515 , nearly $25 \%$ of the 2,257 samples in those scenes, as the training data in Stage-2. Compared with previous fully supervised algorithms which leverage all 951 exhaustively annotated scenes with 2, 257 pedestrian samples, we use far less and weaker supervision. To reduce false negative responses and speed up the OS-NMS process, following [7], we set the $x, z$ range of the searching region for pedestrians as $[(-20,20) \mathrm{m},(0,48) \mathrm{m}]$, respectively.

- Pseudo Foreground Ground-Truth Generation. We directly use a cylinder (with $0.4 \mathrm{~m}$ radius) to generate pseudo binary masks. This is because, different from vehicles, which are typically presented as elongated rectangles on BEV maps, pedestrians are more like regular squares. For the points falling into click-centered cylinders, the foreground probabilities are set as 1 ; otherwise 0 .

- Cylindrical 3D Proposal Generation. Considering the small size of pedestrians, we generate the cylindrical proposal with a $1 \mathrm{~m}$ radius over the $(x, z)$-plane and the proposals whose center-distances are less than $0.5 \mathrm{~m}$ away 
TABLE 3

Evaluation results ( $\S 6.3$ ) on val set of KITTI $3 \mathrm{D}$ and BEV object detection benchmark (Pedestrian). AP with loU 0.5 threshold is reported.

\begin{tabular}{c|c||c|ccc|cr}
\hline Learning Paradigm & Detector & Modality & Easy & $\begin{array}{c}\text { BEV@0.5 } \\
\text { Moderate }\end{array}$ & Hard & $\begin{array}{c}\text { ED Easy } \\
\text { Mox@0.5 } \\
\text { Moderate }\end{array}$ \\
& \multicolumn{1}{|c||}{ Hard } \\
\hline \hline \\
\hline
\end{tabular}
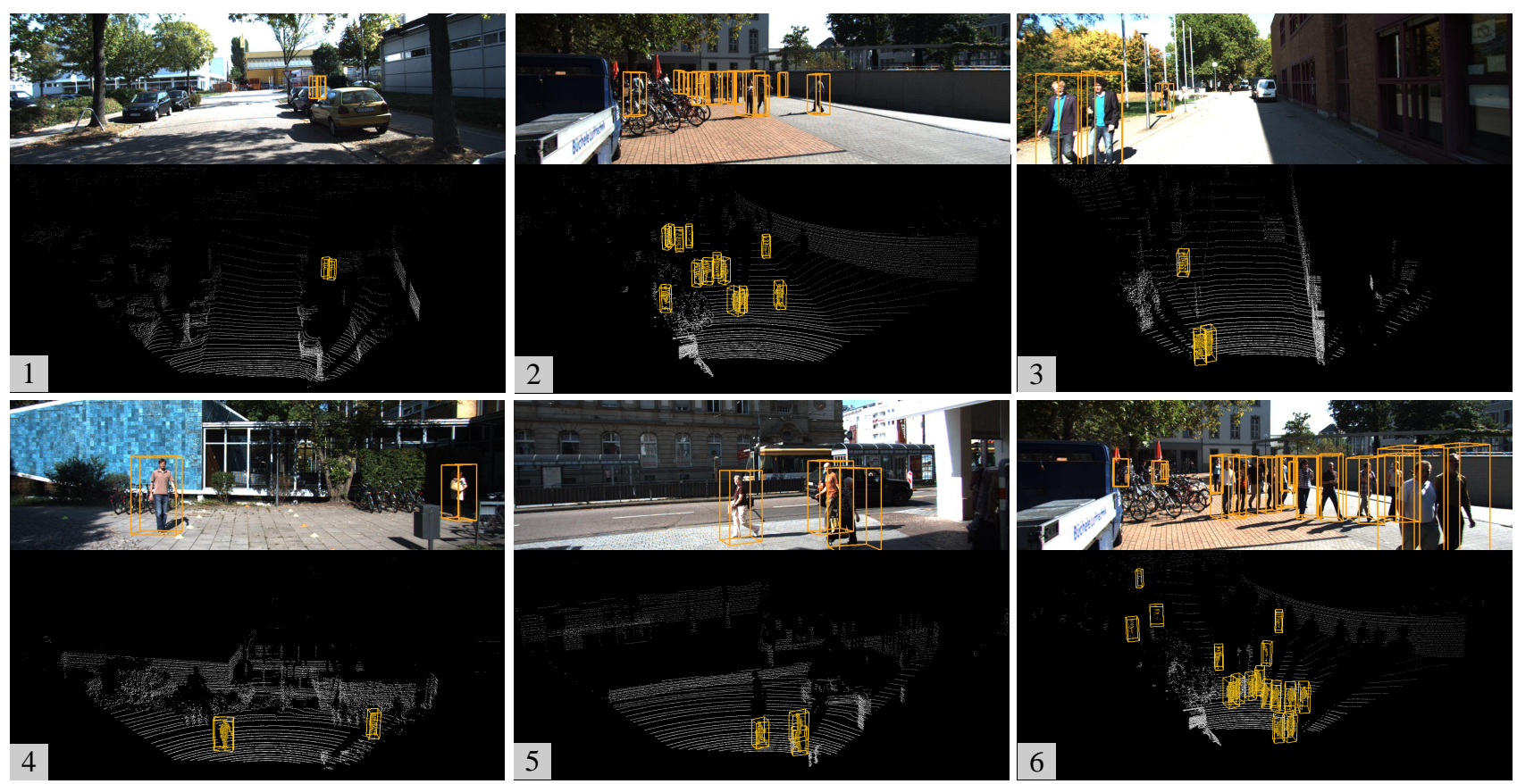

Fig. 8. Qualitative results of 3D object detection (Pedestrian) on KITTI val set (§6.3).

are selected as the training samples for each ground-truth.

- Training. Only one cascade is used, as the predicted boxes are already good enough for the required $0.5 \mathrm{IoU}$ threshold. Consequently, in Stage-2, the whole training process takes $20 \mathrm{~K}$ iterations with a batch-size of 800 .

- Inference. Similar to the Car class, we apply our two-stage network to the input point cloud and use $I o U=0.3$.

Quantitative Results on KITTI val Set. As shown in Table 3, with less and weaker supervision, our method still shows very promising results, even better than current topleading methods. This verifies its good generalizability over different object classes and effectiveness for small objects.

Qualitative Results on KITTI val Set. In Fig. 7, we visualize representative outputs of our model on KITTI val set for the Pedestrian class. As we can see, for simple nonoccluded objects at a reasonable distance (with dense cloud points), our model outputs remarkably accurate 3D boxes (see subfigures 3, 4 and 5). In addition, we are surprised to find that our model can even correctly predict some highly occluded ones (see subfigure 1) and works well in several crowded scenes (subfigures 2 and 6). This proves that our detector not only handles vehicles well, but also adapts to other challenging classes in autonomous driving scenes, under less and easily acquired supervision.

\subsection{Diagnostic Experiment}

We next study the impact of core components of our approach and influence of annotation cost on our final performance. As the ground-truth for KITTI test set is not available and access to the test server is limited, ablation studies are performed over the val set, Car class.

Influence of Different Training Data Sampling Strategies. To facilitate future studies, we directly use the first 500 scenes with weakly annotated BEV maps in KITTI train set as well as $25 \%$ of precisely annotated vehicle instances in the 500 scenes as our default training setting (see §6.2). Due to the use of a small number of training samples, we investigate the impacts of different training data sampling strategies to the final performance. Specifically, we consider diverse training setups, i.e., use No. 1-500, No. 501-1000, 
TABLE 5

Ablation studies ( $\$ 6.4)$ on the efficacy of core model components and impacts of annotation qualities and quantities. Results are reported on va 1 set of KITTI 3D and BEV object detection benchmark (Car), measured by AP with loU 0.7 threshold.

\begin{tabular}{|c|c|c|c|c|c|c|c|c|}
\hline \multicolumn{2}{|l|}{ Aspects } & Training Setting & \multicolumn{3}{|c|}{ BEV@0.7 } & \multicolumn{2}{|c|}{ 3D Box@0.7 } & Hard \\
\hline \multicolumn{2}{|c|}{$\begin{array}{l}\text { Full model (IoU scoring head } \\
+3 \text {-step cascaded refinement) }\end{array}$} & \multirow{5}{*}{$\begin{array}{l}\text { first } 500 \text { weakly labeled scenes } \\
+25 \% \text { precisely annotated instances }\end{array}$} & 88.95 & 85.83 & 85.03 & 85.04 & 75.94 & 74.38 \\
\hline \multirow{3}{*}{ Cascaded refinement } & 1-step & & 83.08 & 75.32 & 75.33 & 68.32 & 61.97 & 62.03 \\
\hline & 2-step & & 88.56 & 84.99 & 84.74 & 84.04 & 75.10 & 73.29 \\
\hline & 4-step & & 88.44 & 84.17 & 78.13 & 84.30 & 74.53 & 72.67 \\
\hline Classification head & 3-step & & 88.02 & 84.31 & 83.80 & 84.44 & 74.99 & 73.85 \\
\hline \multicolumn{2}{|c|}{$\begin{array}{c}\text { More precisely } \\
\text { annotated BEV maps }\end{array}$} & $\begin{array}{l}\text { first } 500 \text { more precisely and weakly labeled scenes } \\
+25 \% \text { precisely annotated instances }\end{array}$ & 89.19 & 85.41 & 85.57 & 86.36 & 76.07 & 74.42 \\
\hline \multirow{3}{*}{\multicolumn{2}{|c|}{ More training data }} & $\begin{array}{c}\text { 3,712 weakly labeled scenes } \\
+25 \% \text { precisely annotated instances }\end{array}$ & 89.40 & 87.88 & 86.19 & 86.54 & 77.02 & 75.49 \\
\hline & & $\begin{array}{l}\text { first } 1,852 \text { weakly labeled scenes } \\
+25 \% \text { precisely annotated instances }\end{array}$ & 89.61 & 86.85 & 86.03 & 87.62 & 77.67 & 76.98 \\
\hline & & $\begin{array}{l}\text { 3,712 weakly labeled scenes } \\
+25 \% \text { precisely annotated instances }\end{array}$ & 89.71 & 87.62 & 86.80 & 87.98 & 78.16 & 77.53 \\
\hline
\end{tabular}

TABLE 4

Ablation study ( $\$ 6.4)$ on the impacts of different training data sampling strategies. Results are reported on val set of KITTI 3D and BEV object detection benchmark (Car), using AP with loU 0.7 threshold.

\begin{tabular}{r||ccc|ccc}
\hline Avg. score & Easy & $\begin{array}{c}\text { BEV@0.7 } \\
\text { Moderate }\end{array}$ & Hard & Easy & $\begin{array}{c}\text { 3D Box@0.7 } \\
\text { Moderate }\end{array}$ & Hard \\
\hline \hline Ours & $88.79_{ \pm 0.84}$ & $85.74_{ \pm 0.57}$ & $85.09_{ \pm 0.58}$ & $85.17_{ \pm 0.87}$ & $75.83_{ \pm 0.69}$ & $74.42_{ \pm 0.74}$ \\
\hline
\end{tabular}

No. 1001-1500, No. 1501-2000, No. 2001-2500, No. 25013000, and last 500 scenes, as well as randomly sampling 500 scenes, from KITTI train set, to train our model, respectively. Note that, for these training settings, only weak BEV annotations and $25 \%$ of the precisely annotated vehicle instances are used. Table 4 reports the average performance with variances over the eight training settings. As seen, the performance changes raised by different training data sampling strategies are negligible.

Cascaded Inference. In $\S 4.2$, the proposal refinement is performed in a coarse-to-fine, cascaded manner. To verify the effectiveness of such a design, we report the performance with different inference steps. As seen, the performance is significantly improved by adding a second step, and becomes stable after three steps. This is because, when using $>3$ cascades, the benefit from the improved learning capacity is marginal, while the negative influence of over-fitting becomes dominant (especially considering we use weaker and fewer annotations). Considering the model complexity and performance, we choose a three-step design as our default setting for the Stage-2 module.

IoU-based Confidence Prediction. For detection confidence estimation, we let our model to predict the IoU score through Eq. 7. To investigate such strategy, we conduct an experiment in which we train our model to predict the classification confidence as the detection confidence, which is widely used in previous 3D detectors [7], [16]. From Table 5 we can observe performance drop after replacing our IoU scoring head with the classification head.

Robustness to Inaccurate BEV Annotations. As discussed in $\S 3, \mathrm{BEV}$ center-click annotations yield about $0.25 \mathrm{~m}$ and $0.75 \mathrm{~m}$ average errors over the $x$ - and $z$-axes, respectively. To examine our robustness to inaccurate BEV annotations, we retrain our model with precise BEV labels inferred from KITTI 3D ground-truths. As shown in Table 5, only marginal improvements can be observed, verifying that our model is not sensitive to the noise in BEV annotations.

More Training Data. To demonstrate the potential of our weakly supervised 3D object detection scheme, we probe the upper bound by training on additional data. As evidenced in the last three rows in Table 5, with more training data, gradual performance boosts can indeed be achieved.

\subsection{Performance as An Annotation Tool}

Our model, once trained, can be applied as a 3D annotator. It only consumes a portion of KITTI train set, allowing its potential in assisting annotation to be explored. Due to its specific network architecture designs and BEV centerclick supervision guided learning paradigm, it supports both automatic and active annotation modes.

Automatic Annotation Mode. For a given scene, it is straightforward to use our model's predictions as annotations, resulting in a fully automatic working mode. In such a setting, our method takes around $0.1 \mathrm{~s}$ per vehicle instance annotation. Previous 3D detection methods can also work as automatic annotators in this way. However, as they are typically trained with the whole KITTI train set, it is hard to examine their annotation quality.

Active Annotation Mode. Limited human supervision is involved in this setting. Human annotators first click object centers in the BEV maps, following the labeling strategy detailed in $\S 3$. Then, for each clicked center, 25 points are uniformly sampled from the surrounding $0.4 \mathrm{~m} \times 0.4 \mathrm{~m}$ region ( $0.1 \mathrm{~m}$ interval). These points are used as the centers of cylindrical proposals and the foreground masks around them are generated according to Eq. 1. Then, we use our Stage- 2 model to predict the cuboids, from which the one with largest confidence score is selected as the final annotation. About $2.6 \mathrm{~s}$ are needed for annotating each vehicle instance in our active annotation mode, including $2.5 \mathrm{~s}$ for human center-click labelling (discussed in §3).

Annotation Quality. Table 6 reports the evaluation results for our annotation quality on KITTI val set (Car). Two previous annotation methods [48], [49] are also included. [48] is a fully supervised deep learning annotator, trained with the 
TABLE 6

Annotation quality ( $(6.5)$ on val set of KITTI $3 \mathrm{D}$ and BEV object detection benchmark (Car). APs with loU 0.5 and 0.7 thresholds are reported.

\begin{tabular}{|c|c|c|c|c|c|c|c|c|c|c|c|c|c|c|c|}
\hline Learning & \multirow{2}{*}{ Method } & \multirow{2}{*}{ Mode } & \multirow{2}{*}{$\begin{array}{c}\text { Speed } \\
\text { (sec./inst.) }\end{array}$} & \multicolumn{3}{|c|}{ BEV@0.5 } & \multicolumn{3}{|c|}{ 3D Box@0.5 } & \multicolumn{3}{|c|}{$\overline{\text { BEV@0.7 }}$} & \multicolumn{3}{|c|}{ 3D Box@0.7 } \\
\hline Paradigm & & & & Easy & Moderate & Hard & Easy & Moderate & Hard & Easy & Moderate & Hard & Easy & Moderate & Hard \\
\hline \multicolumn{16}{|c|}{ Trained with the whole KITTI train set: 3,712 well-labeled scenes with 15,654 vehicle instances } \\
\hline Fully Supervised & [48] & Active & 3.8 & - & - & - & - & - & 88.33 & - & - & - & - & - & - \\
\hline \multicolumn{16}{|c|}{ Trained with the whole KITTI train/val set: 7,481 scenes (implicitly using 2D instance segmentation annotations) } \\
\hline Fully-Supervised & [49] & Auto & 8.0 & 80.70 & 63.36 & 52.47 & 63.39 & 44.79 & 37.47 & - & - & - & - & - & - \\
\hline \multicolumn{16}{|c|}{ Trained with a part of KITTI train set: 500 weakly labeled scenes + 534 precisely annotated instances } \\
\hline \multirow{2}{*}{ Weakly Supervised } & \multirow{2}{*}{ Ours } & Auto & 0.1 & 96.46 & 89.35 & 88.97 & 96.34 & 89.44 & 88.95 & 90.21 & 88.84 & 86.58 & 88.79 & 78.80 & 77.59 \\
\hline & & Active & 2.6 & 99.99 & 99.94 & 99.95 & 99.91 & 90.88 & 90.86 & 98.66 & 90.06 & 89.89 & 89.38 & 86.88 & 79.18 \\
\hline
\end{tabular}

TABLE 7

Performance comparison ( $\S 6.5)$ of 3D object detectors PointRCNN [16] and PointPillars [7] trained on the true KITTI labels vs. labels generated by our annotators in different working modes (i.e., automatic and active). Results are reported on val set of KITTI 3D and BEV object detection benchmark (Car), measured by AP with loU 0.7 threshold.

\begin{tabular}{|c|c|c|c|c|c|c|c|}
\hline Detector & Annotation Source & Easy & $\begin{array}{c}\text { BEV@0.7 } \\
\text { Moderate }\end{array}$ & Hard & Easy & $\begin{array}{c}\text { 3D Box@0.7 } \\
\text { Moderate }\end{array}$ & Hard \\
\hline PointRCNN [16] & $\begin{array}{c}\text { Manual (original) } \\
\text { Automatic (ours) } \\
\text { Active (ours) }\end{array}$ & $\begin{array}{l}90.21 \\
88.49 \\
89.15 \\
\end{array}$ & $\begin{array}{l}87.89 \\
86.24 \\
86.81 \\
\end{array}$ & $\begin{array}{l}85.51 \\
84.96 \\
85.49 \\
\end{array}$ & $\begin{array}{l}88.45 \\
83.69 \\
85.63 \\
\end{array}$ & $\begin{array}{l}77.67 \\
75.05 \\
76.53\end{array}$ & $\begin{array}{l}76.30 \\
73.85 \\
75.47\end{array}$ \\
\hline PointPillars [7] & $\begin{array}{c}\text { Manual (original) } \\
\text { Automatic (ours) } \\
\text { Active (ours) }\end{array}$ & $\begin{array}{l}89.64 \\
89.12 \\
89.54\end{array}$ & $\begin{array}{l}86.46 \\
86.13 \\
86.24\end{array}$ & $\begin{array}{l}84.22 \\
84.15 \\
84.17\end{array}$ & $\begin{array}{l}85.31 \\
85.21 \\
85.28\end{array}$ & $\begin{array}{l}76.07 \\
74.90 \\
75.69\end{array}$ & $\begin{array}{l}69.76 \\
69.00 \\
69.15\end{array}$ \\
\hline
\end{tabular}

whole KITTI train set. It only works as an active model, where humans are required to provide object anchor clicks. [49] requires synthetic data for training and relies on MASKRCNN [59], so it implicitly uses 2D instance segmentation annotations. It works in an automatic mode. The scores of these models are taken from the literature. Following their settings [48], [49], 0.5 IoU criterion is adapted. For providing a more comprehensive evaluation of our model, we also report the annotation performance with 0.7 IoU threshold. As we can see, our model produces high-quality annotations, especially in the active mode. Our annotations are clearly more accurate than [48], [49], with much less and weaker supervision. Considering our fast annotation speed (0.1-2.6 s per instance), the results are very significant.

Suitability for 3D Object Detection. To investigate the suitability of our annotations for 3D object detection, we use our relabeled KITTI train set (Car) to re-train PointPillars [7] and PointRCNN [16], which show leading performance with released implementations. During training, we use their original settings and do not change any hyperparameters. From Table 7, we can observe that the two methods only suffer from small performance drops (at most $5 \%$ ) when using our automatically generated labels. More remarkably, concerning the applicability of the annotations generated under our active mode, the re-trained detectors even performed over $96.8 \%$ of the true labels.

Qualitative Annotation Results. Fig. 9 visualizes some annotation results generated under automatic and active modes. As can be observed, in most cases our automatic model can produce high-quality annotations. When our model works in the active mode, human annotators can place extra center-clicks, thus the inferior or missing predictions can be corrected. In addition, with the help of the weak human supervision, better proposals can be generated around the click points, leading to improved predictions.

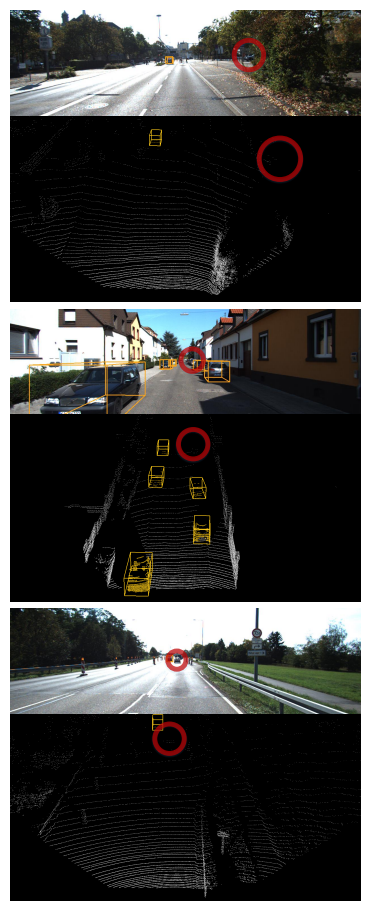

Automatic Mode

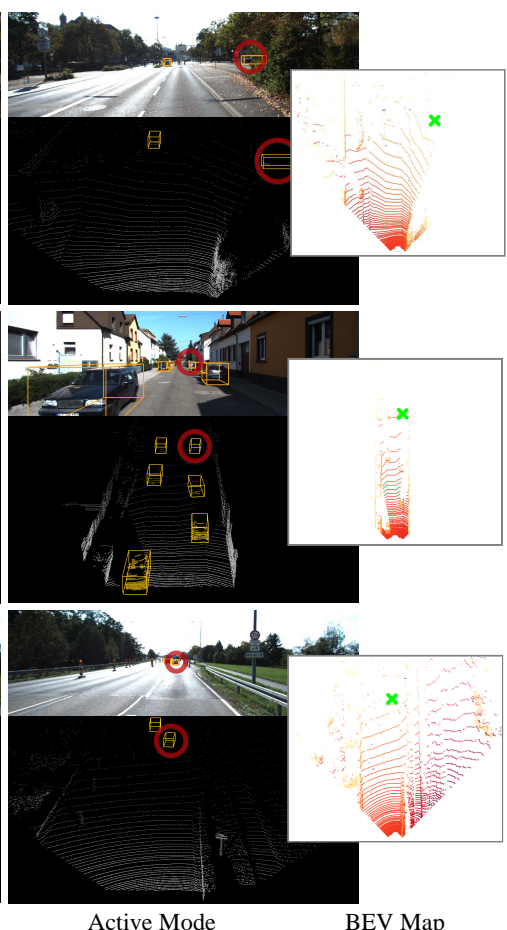

Active Mode
Fig. 9. Annotation results for 3D object detection (Car) on KITTI val set (§6.5). The improved annotations are highlighted by red circles.

\subsection{Failure Cases and Future Work}

Though our predictions are typically accurate, our detector still faces difficulties in some challenging scenarios. Fig. 10 summarizes some common failure cases for 3D vehicle detection. The first type of common mistakes is caused by heavy occlusions, For example, the vehicle in subfigure 1, highlighted in the red circle, is predicted with the wrong height. Leveraging more contextual information may be 

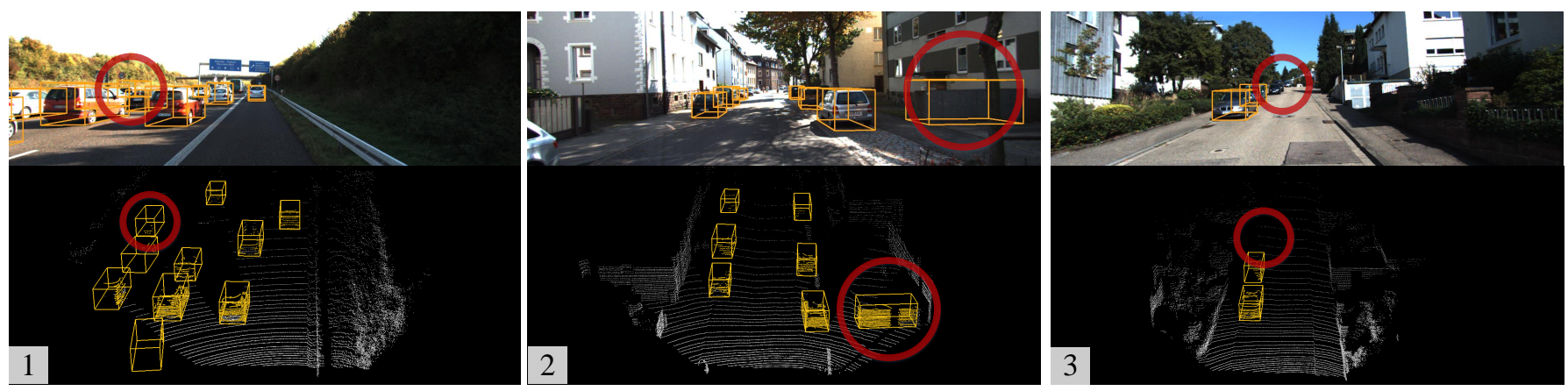

Fig. 10. Failure cases of 3D car detection on KITTI val set (§6.6). The inaccurate predictions are highlighted by red circles.
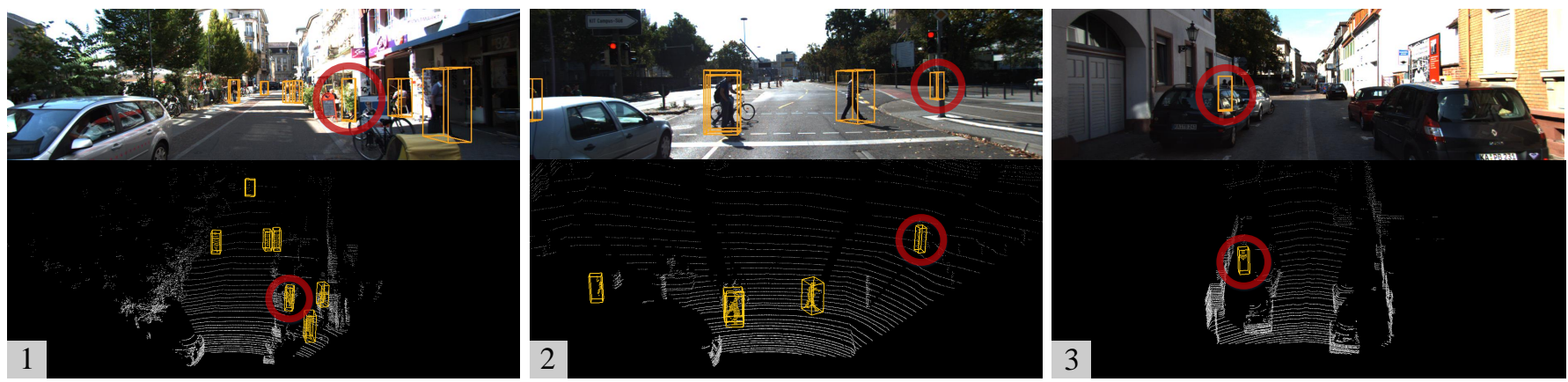

Fig. 11. Failure cases of 3D pedestrian detection on KITTI val set (§6.6). The inaccurate predictions are highlighted by red circles.

helpful. The second type of challenges is brought out by some background obstacles being similar to the target class, like the large box in subfigure 2, which has a similar shape to the vehicle in the point cloud. Third, for some cases where the foreground points are extremely sparse, our model finds it hard to make accurate predictions. Subfigure 3 shows a typical example, there are very few points for the highlighted vehicles due to the hillside occlusion. The last two problems highlight the general limitations of LiDAR based methods; considering texture and optical information should helpful. In the future, we will therefore focus on addressing these issues.

Detecting pedestrians is more challenging and easily leads to similar lapses (see Fig. 11). Our model is occasionally confused by cylindrical obstacles such as the plant in subfigure 1 and the pole in subfigure 2, which are false positives. In subfigure 3 , the two pedestrians are very close and highly occluded, causing our output to merge them together. The above challenges also indicate possible directions for future research.

In addition to addressing the above-mentioned issues, we plan to continue our work in the following directions. First, more diverse supervisory signals can be mined from the inherent structures of 3D data, such as the correspondences between LiDAR and visual cameras, as well as the strong correlations between point data from adjacent frames. Second, more efficient learning protocols can be explored, such as self-/semi-/mixed-supervised learning techniques, to reduce annotation costs and benefit $3 \mathrm{D} \mathrm{ob}-$ ject pattern modeling, thus further facilitating a variety of downstream 3D applications. Third, different kinds of annotation strategies can be developed. Currently, 2D data annotation has achieved great advances and different anno- tation strategies are actively being explored, such as centerclick, extreme-point, and scribbles. However, 3D annotation has received far less attention, even though it is much more time-consuming, harder and also of great value. One straightforward way of extending our annotation approach is to explore both positive and negative click annotations.

\section{Conclusion}

This work has made an early attempt to train a 3D object detector using limited and weak supervision. We addressed the labeling challenge by introducing a quick BEV centerclick annotation strategy and demonstrated using the weak BEV supervision in conjunction with a few precisely annotated 3D instances suffices to train a competitive model. We also detailed how to adapt our trained detector as an easily accessible annotation system, which can reduce human labeling burden and output high-quality annotations. We presented extensive comparative evaluations of our weakly supervised 3D object detector on the classic KITTI dataset and proved its capability of significantly reducing the required scale and quality of supervision. Though impressive results were obtained, there is still large room for further improvement. Given the massive number of algorithmic breakthroughs over the past few years, we can expect a flurry of innovation towards this promising direction.

\section{ACKNOWLEDGEMENTS}

This work was supported by the Natural Science Foundation of China (No. 61773062), CCF-Baidu Open Fund and Zhejiang Lab's Open Fund (No. 2020AA3AB14). 


\section{REFERENCES}

[1] Q. Meng, W. Wang, T. Zhou, J. Shen, L. Van Gool, and D. Dai, "Weakly supervised 3d object detection from lidar point cloud," in Proc. Eur. Conf. Comput. Vis., 2020, pp. 515-531.

[2] X. Chen, K. Kundu, Z. Zhang, H. Ma, S. Fidler, and R. Urtasun, "Monocular 3D object detection for autonomous driving," in Proc. IEEE Conf. Comput. Vis. Pattern Recognit., 2016, pp. 2147-2156.

[3] F. Chabot, M. Chaouch, J. Rabarisoa, C. Teuliere, and T. Chateau, "Deep MANTA: A coarse-to-fine many-task network for joint 2D and 3D vehicle analysis from monocular image," in Proc. IEEE Conf. Comput. Vis. Pattern Recognit., 2017, pp. 1827-1836.

[4] X. Chen, K. Kundu, Y. Zhu, A. G. Berneshawi, H. Ma, S. Fidler, and R. Urtasun, "3D object proposals for accurate object class detection," in Proc. Advances Neural Inf. Process. Syst., 2015, pp. $424-432$.

[5] B. Yang, W. Luo, and R. Urtasun, "Pixor: Real-time 3D object detection from point clouds," in Proc. IEEE Conf. Comput. Vis. Pattern Recognit., 2018, pp. 7652-7660.

[6] Y. Chen, S. Liu, X. Shen, and J. Jia, "Fast point R-CNN," in Proc. IEEE Int. Conf. Comput. Vis., 2019, pp. 9774-9783.

[7] A. H. Lang, S. Vora, H. Caesar, L. Zhou, J. Yang, and O. Beijbom, "Pointpillars: Fast encoders for object detection from point clouds," in Proc. IEEE Conf. Comput. Vis. Pattern Recognit., 2019, pp. 12697-12705.

[8] C. R. Qi, O. Litany, K. He, and L. J. Guibas, "Deep hough voting for 3d object detection in point clouds," in Proc. IEEE Int. Conf. Comput. Vis., 2019, pp. 9277-9286.

[9] X. Chen, H. Ma, J. Wan, B. Li, and T. Xia, "Multi-view 3D object detection network for autonomous driving," in Proc. IEEE Conf. Comput. Vis. Pattern Recognit., 2017, pp. 1907-1915.

[10] C. R. Qi, X. Chen, O. Litany, and L. J. Guibas, "Imvotenet: Boosting $3 \mathrm{~d}$ object detection in point clouds with image votes," in Proc. IEEE Conf. Comput. Vis. Pattern Recognit., 2020, pp. 4404-4413.

[11] D. Maturana and S. Scherer, "Voxnet: A 3D convolutional neural network for real-time object recognition," in IEEE Trans. Intell. Robots and Systems, 2015, pp. 922-928.

[12] Z. Wu, S. Song, A. Khosla, F. Yu, L. Zhang, X. Tang, and J. Xiao, "3D shapenets: A deep representation for volumetric shapes," in Proc. IEEE Conf. Comput. Vis. Pattern Recognit., 2015, pp. 1912-1920.

[13] C. R. Qi, H. Su, K. Mo, and L. J. Guibas, "Pointnet: Deep learning on point sets for 3D classification and segmentation," in Proc. IEEE Conf. Comput. Vis. Pattern Recognit., 2017, pp. 652-660.

[14] B. Li, T. Zhang, and T. Xia, "Vehicle detection from 3D Lidar using fully convolutional network," in Robotics: Science and Systems, 2016, pp. 1513-1518.

[15] Y. Yan, Y. Mao, and B. Li, "Second: Sparsely embedded convolutional detection," Sensors, vol. 18, no. 10, p. 3337, 2018.

[16] S. Shi, X. Wang, and H. Li, "PointRCNN: 3D object proposal generation and detection from point cloud," in Proc. IEEE Conf. Comput. Vis. Pattern Recognit., 2019, pp. 770-779.

[17] Y. Zhou and O. Tuzel, "Voxelnet: End-to-end learning for point cloud based 3D object detection," in Proc. IEEE Conf. Comput. Vis. Pattern Recognit., 2018, pp. 4490-4499.

[18] Z. Yang, Y. Sun, S. Liu, X. Shen, and J. Jia, "STD: Sparse-to-dense 3D object detector for point cloud," in Proc. IEEE Int. Conf. Comput. Vis., 2019, pp. 1951-1960.

[19] A. Geiger, P. Lenz, and R. Urtasun, "Are we ready for autonomous driving? the KITTI vision benchmark suite," in Proc. IEEE Conf. Comput. Vis. Pattern Recognit., 2012, pp. 3354-3361.

[20] Y. Xiang, W. Choi, Y. Lin, and S. Savarese, "Data-driven 3D voxel patterns for object category recognition," in Proc. IEEE Conf. Comput. Vis. Pattern Recognit., 2015, pp. 1903-1911.

[21] J. Xie, Z. Zheng, R. Gao, W. Wang, S.-C. Zhu, and Y. Nian Wu, "Learning descriptor networks for $3 \mathrm{~d}$ shape synthesis and analysis," in Proc. IEEE Conf. Comput. Vis. Pattern Recognit., 2018, pp. 8629-8638.

[22] H. Su, S. Maji, E. Kalogerakis, and E. Learned-Miller, "Multi-view convolutional neural networks for 3D shape recognition," in Proc. IEEE Int. Conf. Comput. Vis., 2015, pp. 945-953.

[23] C. R. Qi, H. Su, M. Niessner, A. Dai, M. Yan, and L. J. Guibas, "Volumetric and multi-view cnns for object classification on 3D data," in Proc. IEEE Conf. Comput. Vis. Pattern Recognit., 2016, pp. 5648-5656.

[24] D. Rethage, J. Wald, J. Sturm, N. Navab, and F. Tombari, "Fullyconvolutional point networks for large-scale point clouds," in Proc. Eur. Conf. Comput. Vis., 2018, pp. 596-611.
[25] C. R. Qi, L. Yi, H. Su, and L. J. Guibas, "Pointnet++: Deep hierarchical feature learning on point sets in a metric space," in Proc. Advances Neural Inf. Process. Syst., 2017, pp. 5099-5108.

[26] C. R. Qi, W. Liu, C. Wu, H. Su, and L. J. Guibas, "Frustum pointnets for 3D object detection from RGB-D data," in Proc. IEEE Conf. Comput. Vis. Pattern Recognit., 2018, pp. 918-927.

[27] A. Mousavian, D. Anguelov, J. Flynn, and J. Kosecka, "3D bounding box estimation using deep learning and geometry," in Proc. IEEE Conf. Comput. Vis. Pattern Recognit., 2017, pp. 5632-5640.

[28] B. Li, W. Ouyang, L. Sheng, X. Zeng, and X. Wang, "GS3D: An efficient 3D object detection framework for autonomous driving," in Proc. IEEE Conf. Comput. Vis. Pattern Recognit., 2019, pp. 10191028.

[29] R. Mottaghi, Y. Xiang, and S. Savarese, "A coarse-to-fine model for 3D pose estimation and sub-category recognition," in Proc. IEEE Conf. Comput. Vis. Pattern Recognit., 2015, pp. 418-426.

[30] Z. Yang, Y. Sun, S. Liu, and J. Jia, “3DSSD: Point-based 3d single stage object detector," in Proc. IEEE Conf. Comput. Vis. Pattern Recognit., 2020, pp. 11040-11048.

[31] M. Liang, B. Yang, S. Wang, and R. Urtasun, "Deep continuous fusion for multi-sensor 3D object detection," in Proc. Eur. Conf. Comput. Vis., 2018, pp. 663-678.

[32] M. Liang, B. Yang, Y. Chen, R. Hu, and R. Urtasun, "Multi-task multi-sensor fusion for 3D object detection," in Proc. IEEE Conf. Comput. Vis. Pattern Recognit., 2019, pp. 7337-7345.

[33] A. Bearman, O. Russakovsky, V. Ferrari, and L. Fei-Fei, "What's the point: Semantic segmentation with point supervision," in Proc. Eur. Conf. Comput. Vis., 2016, pp. 549-565.

[34] D. P. Papadopoulos, J. R. Uijlings, F. Keller, and V. Ferrari, “Training object class detectors with click supervision," in Proc. IEEE Conf. Comput. Vis. Pattern Recognit., 2017, pp. 6374-6383.

[35] K.-K. Maninis, S. Caelles, J. Pont-Tuset, and L. Van Gool, “Deep extreme cut: From extreme points to object segmentation," in Proc. IEEE Conf. Comput. Vis. Pattern Recognit., 2018, pp. 619-625.

[36] D. P. Papadopoulos, J. R. Uijlings, F. Keller, and V. Ferrari, "Extreme clicking for efficient object annotation," in Proc. IEEE Int. Conf. Comput. Vis., 2017, pp. 4930-4939.

[37] R. Benenson, S. Popov, and V. Ferrari, "Large-scale interactive object segmentation with human annotators," in Proc. IEEE Conf. Comput. Vis. Pattern Recognit., 2019, pp. 11700-11 709.

[38] A. Kar, A. Prakash, M.-Y. Liu, E. Cameracci, J. Yuan, M. Rusiniak, D. Acuna, A. Torralba, and S. Fidler, "Meta-sim: Learning to generate synthetic datasets," in Proc. IEEE Int. Conf. Comput. Vis., 2019, pp. 4551-4560.

[39] D. Stutz and A. Geiger, "Learning 3d shape completion from laser scan data with weak supervision," in Proc. IEEE Conf. Comput. Vis. Pattern Recognit., 2018, pp. 1955-1964.

[40] J. Gwak, C. B. Choy, M. Chandraker, A. Garg, and S. Savarese, "Weakly supervised $3 \mathrm{~d}$ reconstruction with adversarial constraint," in International Conference on 3D Vision, 2017, pp. 263-272.

[41] X. Xu and G. H. Lee, "Weakly supervised semantic point cloud segmentation: Towards 10x fewer labels," in Proc. IEEE Conf. Comput. Vis. Pattern Recognit., 2020, pp. 13 706-13715.

[42] N. Mayer, E. Ilg, P. Hausser, P. Fischer, D. Cremers, A. Dosovitskiy, and T. Brox, "A large dataset to train convolutional networks for disparity, optical flow, and scene flow estimation," in Proc. IEEE Conf. Comput. Vis. Pattern Recognit., 2016, pp. 4040-4048.

[43] Q. Ma, J. Yang, A. Ranjan, S. Pujades, G. Pons-Moll, S. Tang, and M. J. Black, "Learning to dress 3d people in generative clothing," in Proc. IEEE Conf. Comput. Vis. Pattern Recognit., 2020, pp. 64696478.

[44] N. Zhao, T.-S. Chua, and G. H. Lee, "SESS: Self-ensembling semisupervised $3 \mathrm{~d}$ object detection," in Proc. IEEE Conf. Comput. Vis. Pattern Recognit., 2020, pp. 11079-11 087.

[45] H. Wang, Y. Cong, O. Litany, Y. Gao, and L. J. Guibas, "3DIoUMatch: Leveraging iou prediction for semi-supervised $3 \mathrm{~d}$ object detection," arXiv preprint arXiv:2012.04355, 2020.

[46] S. Xie, J. Gu, D. Guo, C. R. Qi, L. Guibas, and O. Litany, "PointContrast: Unsupervised pre-training for $3 \mathrm{~d}$ point cloud understanding," in Proc. Eur. Conf. Comput. Vis., 2020, pp. 574-591.

[47] Y. S. Tang and G. H. Lee, "Transferable semi-supervised 3d object detection from rgb-d data," in Proc. IEEE Int. Conf. Comput. Vis., 2019, pp. 1931-1940.

[48] J. Lee, S. Walsh, A. Harakeh, and S. L. Waslander, "Leveraging pre-trained 3D object detection models for fast ground truth generation," in The IEEE Intelligent Transportation Systems Conference, 2018, pp. 2504-2510. 
[49] S. Zakharov, W. Kehl, A. Bhargava, and A. Gaidon, "Autolabeling 3D objects with differentiable rendering of SDF shape priors," in Proc. IEEE Conf. Comput. Vis. Pattern Recognit., 2019, pp. 12224 12233.

[50] J. Xie, M. Kiefel, M.-T. Sun, and A. Geiger, "Semantic instance annotation of street scenes by 3D to 2D label transfer," in Proc. IEEE Conf. Comput. Vis. Pattern Recognit., 2016, pp. 3688-3697.

[51] P. Wang, X. Huang, X. Cheng, D. Zhou, Q. Geng, and R. Yang, "The apolloscape open dataset for autonomous driving and its application," IEEE Trans. Pattern Anal. Mach. Intell., pp. 1067-1073, 2019.

[52] S. Song, S. P. Lichtenberg, and J. Xiao, "SUN RGB-D: A RGBD scene understanding benchmark suite," in Proc. IEEE Conf. Comput. Vis. Pattern Recognit., 2015, pp. 567-576.

[53] T.-Y. Lin, P. Goyal, R. Girshick, K. He, and P. Dollar, “Focal loss for dense object detection," in Proc. IEEE Int. Conf. Comput. Vis., 2017, pp. 2980-2988.

[54] Z. Cai and N. Vasconcelos, "Cascade R-CNN: Delving into high quality object detection," in Proc. IEEE Conf. Comput. Vis. Pattern Recognit., 2018, pp. 6154-6162.

[55] K. Chen, J. Pang, J. Wang, Y. Xiong, X. Li, S. Sun, W. Feng, Z. Liu, J. Shi, W. Ouyang et al., "Hybrid task cascade for instance segmentation," in Proc. IEEE Conf. Comput. Vis. Pattern Recognit., 2019, pp. 4974-4983.

[56] T. Zhou, S. Qi, W. Wang, J. Shen, and S.-C. Zhu, "Cascaded parsing of human-object interaction recognition," IEEE Trans. Pattern Anal. Mach. Intell., 2021.

[57] S. Shi, Z. Wang, J. Shi, X. Wang, and H. Li, "From points to parts: $3 \mathrm{~d}$ object detection from point cloud with part-aware and partaggregation network," IEEE Trans. Pattern Anal. Mach. Intell., 2020.

[58] Z. Liu, X. Zhao, T. Huang, R. Hu, Y. Zhou, and X. Bai, "Tanet: Robust $3 \mathrm{~d}$ object detection from point clouds with triple attention," The AAAI Conference on Artificial Intelligence, pp. 11677-11684, 2020.

[59] K. He, G. Gkioxari, P. Dollár, and R. Girshick, "Mask r-cnn," in Proc. IEEE Int. Conf. Comput. Vis., 2017, pp. 2980-2988.

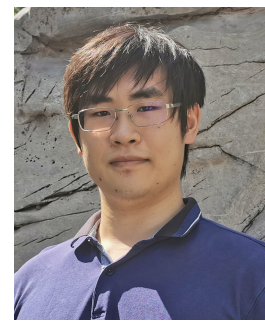

Qinghao Meng received B.S. degree from Beijing Institute of Technology in 2018. Currently he is pursuing Ph.D. degree in Beijing Institute of Technology, advised by Prof. Jianbing Shen. From 2019 to 2020, he was an intern at Inception Institute of Artificial Intelligence, UAE, supervised by Dr. Wenguan Wang. His research interest include 3D computer vision and weakly supervised learning.

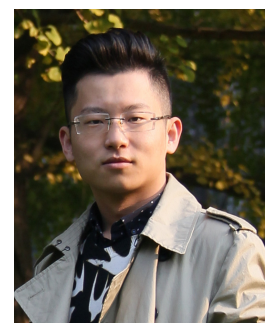

Wenguan Wang received his Ph.D. degree from Beijing Institute of Technology in 2018. He is currently a postdoc scholar at ETH Zurich, Switzerland. From 2016 to 2018, he was a visiting Ph.D. student in University of California, Los Angeles. From 2018 to 2019, he was a senior scientist at Inception Institute of Artificial Intelligence, UAE. His current research interests include computer vision, image processing and deep learning.

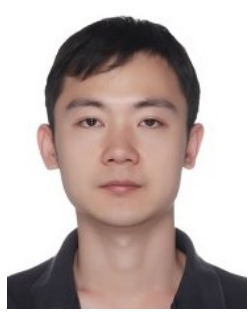

Tianfei Zhou received his Ph.D. degree from Beijing Institute of Technology in 2017. He is currently a postdoc researcher at ETH Zurich, Switzerland. From 2019 to 2020, he was a research associate at Inception Institute of Artificial Intelligence, UAE. His current research interests include human-object interaction recognition, video segmentation and deep learning.

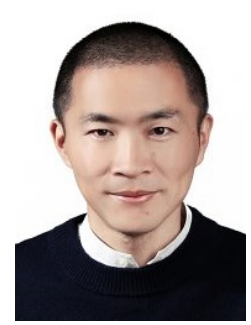

Jianbing Shen (M'11-SM'12) is currently acting as the Lead Scientist with the Inception Institute of Artificial Intelligence, UAE. He is also a Ful Professor with the School of Computer Science, Beijing Institute of Technology. He has published about 100 journal and conference papers. He was awarded the Highly Cited Researcher by Web of Science in 2020. His research interests include computer vision, deep learning, autonomous driving, and medical image analysis. TNNLS and other journals.

He is an Associate Editor of IEEE TIP, IEEE

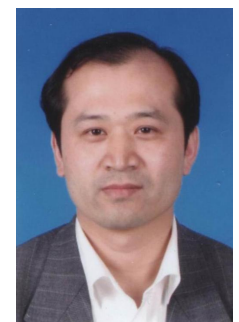

Yunde Jia received the MS and Ph.D. degrees in mechatronics from the Beijing Institute of Technology (BIT), in 1986 and 2000, respectively. $\mathrm{He}$ is currently a professor of computer science with BIT and serves as the director of the Beijing Laboratory of Intelligent Information Technology, School of Computer Science, BIT. He has previously served as the executive dean of the School of Computer Science, BIT, from 2005 to 2008. He was a visiting scientist at Carnegie Mellon University, Pittsburgh, Pennsylvania, from 1995 to 1997, and a Visiting Fellow at the Australian National University, Acton, Australia, in 2011. His current research interests include computer vision, media computing, and intelligent systems.

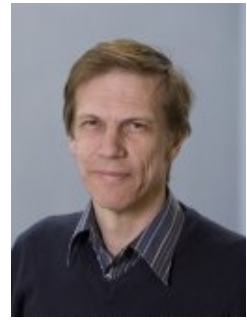

Luc Van Gool received the degree in electromechanical engineering at the Katholieke Universiteit Leuven in 1981. Currently, he is a professor at the Katholieke Universiteit Leuven in Belgium and the ETH in Zurich, Switzerland. He leads computer vision research at both places, and also teaches at both. He has been a program committee member of several major computer vision conferences. His main interests include $3 \mathrm{D}$ reconstruction and modeling, object recognition, tracking, and gesture analysis, and the combination of those. He received several Best Paper awards, won a David Marr Prize and a Koenderink Award, and was nominated Distinguished Researcher by the IEEE Computer Science committee. He is a co-founder of 10 spin-off companies. 